\title{
Folds and Buckles at the Nanoscale: Experimental and Theoretical Investigation of the Bending Properties of Graphene Membranes
}

\author{
Vittorio Morandi, Luca Ortolani, Andrea Migliori, \\ Degli Esposti Boschi Cristian, Emiliano Cadelano, and Luciano Colombo
}

\begin{abstract}
The elastic properties of graphene crystals have been extensively investigated, revealing unique properties in the linear and nonlinear regimes, when the membranes are under either stretching or bending loading conditions. Nevertheless less knowledge has been developed so far on folded graphene membranes and ribbons. It has been recently suggested that fold-induced curvatures, without in-plane strain, can affect the local chemical reactivity, the mechanical properties, and the electron transfer in graphene membranes. This intriguing perspective envisages a materials-by-design approach through the engineering of folding and bending to develop enhanced nano-resonators or nano-electro-mechanical devices. Here we present a novel methodology to investigate the mechanical properties of folded and wrinkled graphene crystals, combining transmission electron microscopy mapping of 3D curvatures and theoretical modeling based on continuum elasticity theory and tight-binding atomistic simulations.
\end{abstract}

Keywords 3D reconstruction . Bending rigidity . Geometric phase analysis . Graphene · Tight binding · Transmission electron microscopy

V. Morandi ( $ه$ ), L. Ortolani, A. Migliori and D.E.B. Cristian CNR-IMM Bologna, via Gobetti 101, I-40129 Bologna, Italy

e-mail: morandi@bo.imm.cnr.it

E. Cadelano and L. Colombo

Department of Physics, University of Cagliari, Cittadella Universitaria, I-09042 Monserrato (Ca), Italy 


\section{Contents}

1 Introduction

2 Mapping Curvature at the Nanoscale

2.1 Geometric Phase Analysis

2.2 Experimental Reconstruction of Bent Graphene Membranes

3 Modeling the Bending Properties of Graphene Membranes: Conceptual Framework

4 Simulation Protocol

4.1 Step 1: Predicting the Shape of a Folded Continuum Membrane

4.2 The Bending Rigidity of Graphene

4.3 Step 2: Predicting the Actual Atomistic Structure of a Folded Graphene Membrane

5 Validation of the Experimental Procedure

6 Conclusions

References

\section{Abbreviations}

$\begin{array}{ll}\text { BLE } & \text { Bilayered edged graphene } \\ \text { CNT } & \text { Carbon nanotube } \\ \text { CVD } & \text { Chemical vapor deposition } \\ \text { DP } & \text { Diffraction pattern } \\ \text { FFT } & \text { Fast Fourier transform } \\ \text { GPA } & \text { Geometric phase analysis } \\ \text { HRTEM } & \text { High resolution transmission electron microscopy } \\ \text { STEM } & \text { Scanning transmission electron microscope } \\ \text { TB } & \text { Tight-binding } \\ \text { TEM } & \text { Transmission electron microscope }\end{array}$

\section{Introduction}

The capabilities of modern low-voltage aberration-corrected TEMs and STEMs, in terms of resolution in imaging and associated spectroscopies, enable the investigation of a wide range of properties of graphene-based materials, with atomic sensitivity and resolution. Among these investigations we can find the morphological aspects (shape, dimensions, thickness), as well as the structural ones (crystalline habits, edges, defects, strain), to the physical and chemical properties (doping, functionalization) of the systems under analysis, [1-5], using these instruments and the related techniques as fundamental tools for the investigation of graphenebased materials $[6,7]$.

In this work we will focus on the structural properties of graphene membranes and, in more detail, on their 3D structure, showing how this can be reconstructed through a combined experimental-theoretical approach taking into account, on one side, geometric phase analysis (GPA) of HREM [8, 9] images, and, on the other, a combination of continuum elasticity theory and atomistic TB simulations. 

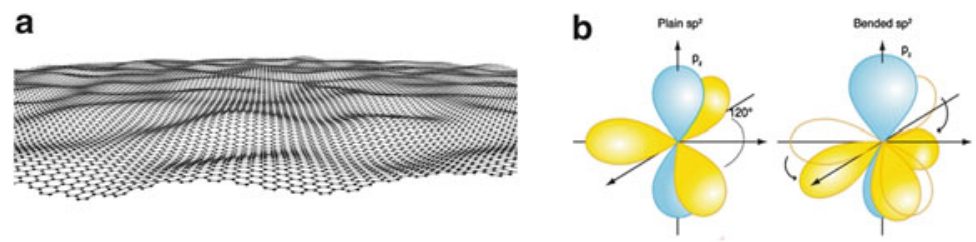

Fig. 1 (a) 3D sketch of an intrinsically bent free-standing graphene flake; (b) graphical representation of the bent $\mathrm{sp}^{2}$ orbitals

\section{Mapping Curvature at the Nanoscale}

The stability of 2D crystals was debated for decades since, according to the so-called Mermin-Wagner theorem, in 2D lattices thermal agitation will induce long wavelength fluctuations which will destroy the long-range crystalline order [10]. The existence of graphene was then debated until the discovery of a full set of $2 \mathrm{D}$ crystals in 2005 [11].

Since the beginning it was clear that the structure of graphene was not perfectly flat; nevertheless nowadays not so much is known on the precise 3D structure of free-standing membranes. Indeed, free-standing graphene exists as a crumpled sheet stabilized by its intrinsic corrugations [12]. Ripples and folds induce curvatures in the $2 \mathrm{D}$ carbon lattice, bending the $\mathrm{sp}^{2}$ sigma bonds and locally modifying the electronic properties of the materials (see Fig. 1).

Graphene membranes, when not supported or suspended, folds, realizing complex structures [13-15]. It is worth noting that whenever the honeycomb lattice bends, its electronic transport properties change, and interesting edge conduction states can be obtained over micrometric lengths. This suggested the possibility to engineer the transport properties of this material by modifying its 3D structure, and strain and bending have been the subject of intense theoretical and experimental studies [16-19]. Recent results indicate that the curvature induced by folding can be at the origin of significant changes of chemical reactivity [20,21] and of the mechanical [22] and charge transport properties [23, 24] of graphene membranes.

The folding of graphene membranes depends on several factors, like lattice orientations, crystal defects, and possibly adsorbed molecules, as well as on the surrounding environment [25-28]. A deeper understanding of the mechanism leading to the bending and the folding of the membranes is still an issue and a deeper understanding of the curvature mechanics in graphene is essential to understand the profound relations between its 3D structure and its properties. In this framework there are two methodologies typically adopted to recover 3D structures at the nanoscale, i.e., electron tomography in the TEM and scanning probe microscopy (SPM).

Unfortunately, neither of these techniques work properly for suspended graphene membranes. Indeed, electron tomography needs to acquire more than 100 images of the same region in different projections to reconstruct its 3D structure successfully [29], a requirement that hardly matches with the high electron beam damage 
a

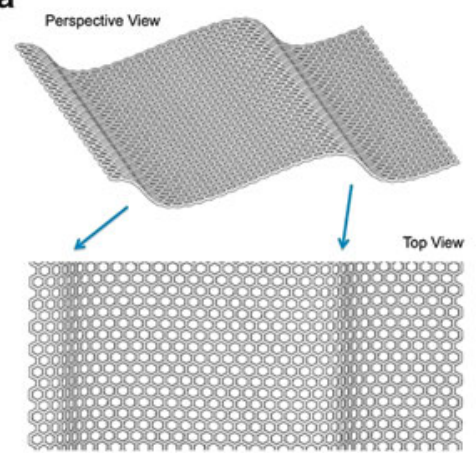

b

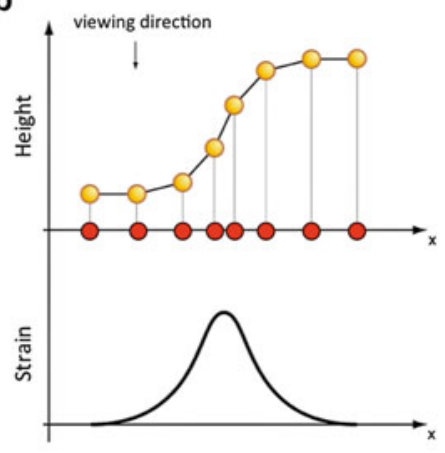

Fig. 2 (a) Schematics of the perspective view and the top view of a bent 2D crystal; (b) 1D sloped atomic chains (yellow circles) with the corresponding projected image on the $x$-axis (red circles) (top) and calculated apparent strain along the $x$-axis (bottom)

sensitivity, even at low energies, of graphene membranes [30]. On the other hand, SPM typically needs supported samples to accomplish the interaction between the probe and the membranes and to avoid, or at least minimize, artifacts due to modification of the structure of the membrane [31].

Here we present a novel method to map 3D deformations in suspended graphene membrane, requiring only one micrograph, and based on the so-called GPA that will be discussed in detail in the following.

The idea behind this is indeed very simple. If we observe in the TEM a wrinkled two-dimensional crystal like that depicted in Fig. 2a, the microscope will provide us with a projected image of the crystal lattice in the direction of the beam. The effect of this projection is that regions of the membrane not perpendicular to the electron beam will appear as compressed. Therefore, from the measurements of these apparent strains in the image of the lattice, in principle one can calculate back the local slope of the flake with respect to the electron beam.

To focus this approach, we can consider a simplified model, such as that represented in Fig. 2b, where, on the top, a one-dimensional chain of atoms showing a height variation is depicted. Despite interatomic distances being kept fixed, in the TEM image we will see the atomic positions projected in the direction of the beam over the $x$-axis, indicated by the red circles. It is worth noting that the distances between the projected positions in the image varies in accordance with the slope of the undulated atomic chain. At the bottom of Fig. $2 \mathrm{~b}$ is shown a profile of the local strain as it would be calculated from the projected atomic positions. Using simple geometry, it is clear that a measure of this apparent strain in the image will provide an immediate measure of the local slope of the undulated chain, thereby providing a complete reconstruction of its two-dimensional structure.

Let us introduce in more detail the principles of image formation in the TEM, as this will be the basis for a complete understanding of the strain recovery techniques. If we consider a crystal illuminated by an electron beam, as in the TEM, each of the 
a

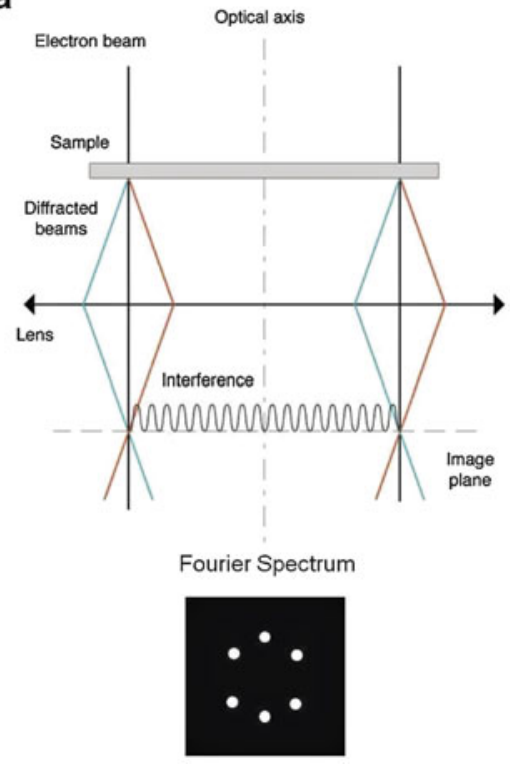

b
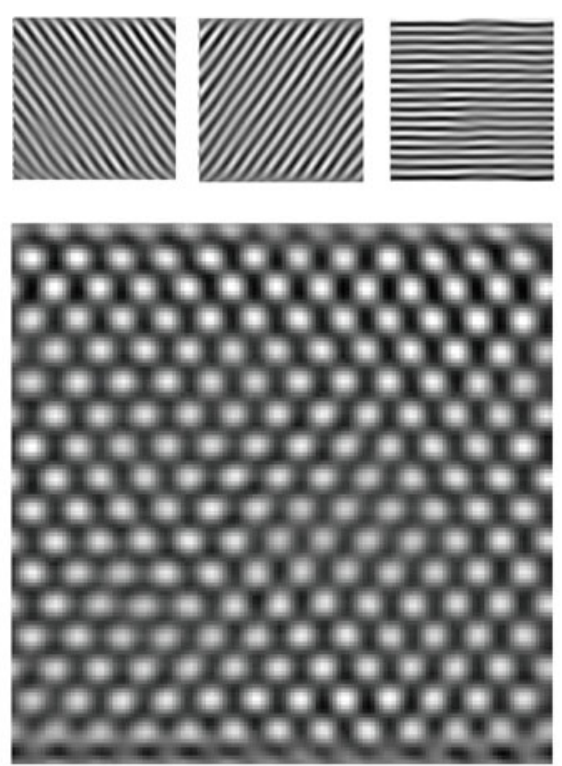

Fig. 3 (a) Schematics of the interference pattern generation on the image plane and typical hexagonal direction pattern of a honeycomb crystal lattice; (b) HREM image formation by the superposition of three interference patterns generated by three different families of planes

crystal planes forming the structure will split the electron beam into multiple beams, the unscattered beam and several diffracted beams, as shown at the top of Fig. 3a. The objective lens of the TEM at high magnification, i.e., in the high resolution imaging mode, is set to bring those diffracted beams to superimpose on the image plane, resulting in an interference pattern, where the fringes follow the direction of the original crystal planes. At the top of Fig. $3 b$ there are reported, as an example, three sets of diffracted wavefronts representing three independent sets of crystal planes.

The results of the superposition of these diffracted wavefronts is shown at the bottom. The interference pattern represents the so-called high resolution electron microscopy image (HREM). In the reciprocal space, like in a diffraction pattern, the fringes appear as couples of symmetric spots, indicating a precise frequency corresponding to the spacing of the fringes, as shown at the bottom of Fig. 3a. A real as well as an apparent deformation of the crystal plane periodicities, like those highlighted in Fig. 2, will result in a deformation of the fringes in the image, and the corresponding information in the reciprocal space will be encoded in the region around the corresponding spot. Therefore, once taking into account the ability to separate the contribution to the modulation of the fringe spacing in an HREM image of the real and of the apparent deformations of the crystal, the problem of mapping undulations and bending of a graphene membrane can be reduced to recovering the strain out of the HREM image. 


\subsection{Geometric Phase Analysis}

GPA is a technique that analyzes the geometric distortions in the HREM image of a crystal lattice by means of Fourier analysis. The reconstructed phase is called geometric to avoid confusion with the electronic phase of the electron wavefront: its meaning and the information it contains on the geometric distortions of the image will be explained and discussed in the following. It is appropriate at this point to introduce some formalism that will facilitate understanding of the underlying mechanism of GPA strain reconstruction.

An HREM micrograph is a 2D image and a vector $\boldsymbol{r}$ can be defined in order to indicate the position of a point. The intensity $I(\boldsymbol{r})$ of the HREM image, as discussed in the previous section, is represented by the superposition of interference fringes created by the various beams diffracted by the sample. We can identify the direction of these beams, and the direction of the relative system of fringes in the image, with the wave-vectors $g$ of the reciprocal space. If we consider the image of a perfect crystal, free from any deformation, its intensity $I(\boldsymbol{r})$ can be expressed as a Fourier series over the frequencies $g$, as

$$
I(\boldsymbol{r})=\sum_{g} I_{g} \mathrm{e}^{2 \pi i g \cdot \boldsymbol{r}},
$$

where $I_{g}$ is a coefficient representing the intensity of the fringe system originating from that particular $g$. In the reciprocal space the Fourier transform of (1) becomes:

$$
\tilde{I}(\boldsymbol{k})=\sum_{g} I_{g} \delta(\boldsymbol{k}-\boldsymbol{g}),
$$

where $\delta$ is the Dirac delta function. For a perfect crystal, the reciprocal space is non-zero only at the positions of the $\boldsymbol{g}$ vectors.

Deformations in the specimen lattice can be introduced using the displacement field $\boldsymbol{u}(\boldsymbol{r})$ with the following transformation [32]:

$$
\boldsymbol{r} \mapsto \boldsymbol{r}-\boldsymbol{u}(\boldsymbol{r})
$$

The effect of this displacement vector field is that the reciprocal lattice directions $g$ are not defined globally for the crystal; instead they are local, depending on the position $\boldsymbol{g}(\boldsymbol{r})$. Then for a deformed crystal (1) becomes

$$
I(\boldsymbol{r})=\sum_{\boldsymbol{g}} I_{g} \mathrm{e}^{2 \pi i \boldsymbol{g} \cdot \boldsymbol{r}} \mathrm{e}^{-2 \pi i \boldsymbol{g} \cdot \boldsymbol{u}(\boldsymbol{r})} .
$$

In real crystals lattice distortions are not the only imperfections present, since we need to take into account thickness variations and, as in the case of graphene 
membranes, possible undulations. All these effects require one to consider the intensity coefficients $I_{g}$ to be the local function $I_{g}(r)$ of the position [8]. If we define the complex functions $H_{g}(\boldsymbol{r})$ as

$$
H_{g}(\boldsymbol{r})=I_{g}(\boldsymbol{r}) \mathrm{e}^{-2 \pi i \boldsymbol{g} \cdot \boldsymbol{u}(\boldsymbol{r})}
$$

then the Fourier transform of (4) becomes

$$
\tilde{I}(\boldsymbol{k})=\sum_{g} \tilde{H}_{g}(\boldsymbol{k}) \otimes \delta(\boldsymbol{k}-\boldsymbol{g})
$$

In the case of a deformed crystal, in the reciprocal space there is now some dispersion of the intensity around the positions of the reciprocal vectors $g$. The information about the deformations in the sample is encoded in the $\tilde{H}_{g}(\boldsymbol{k})$ functions. The amplitude term of these functions will give the modulation in intensity of the interference fringes in the direction of each $g$, while the phase term describes the variations in the inter-fringe spacing around the image area. As already mentioned, this phase term is called geometric phase.

The original HREM digital image is transformed by FFT to its frequency spectrum and the pixels close to a specific $\boldsymbol{g}$ vector are selected using a circular mask. The distance between two nearby $g$ s limit the diameter of the mask, and therefore it limits the resolution of the reconstructed maps, which is usually of the order of a nanometer. The effect of the shape of the mask is beyond the scope of this manuscript and the explicit expression of the mask will be omitted in the following calculations (see [33] for details).

Selecting the region of the reciprocal space around a particular $\boldsymbol{g}$ we are selecting one specific $\tilde{H}_{g}(\boldsymbol{k})$ and setting the origin of the Cartesian reference system to the position of $\boldsymbol{g}$. Inverse FFT will give back the complex image:

$$
\operatorname{FFT}\left(\tilde{H}_{g}(\boldsymbol{k})\right)=H_{g}(\boldsymbol{r})=I_{g}(\boldsymbol{r}) \mathrm{e}^{-2 \pi i \boldsymbol{g} \cdot \boldsymbol{u}(\boldsymbol{r})+\phi_{g}} .
$$

When transforming back an additional phase constant, $\phi_{g}$ emerges. Mathematically the process should recover $H$ back without any additional term, but the pixel nature of the image makes it impossible to determine exactly the position of the $g$, which often lies in a sub-pixel position. This error in the re-centering of the reciprocal space means that a $\delta$-like component is still present and will transform back to a constant phase in real space. The constant phase term $\phi_{g}$ is removed from the reconstructed phase by re-normalizing the background over a reference area of the map [8].

The result of the reconstruction procedure is a complex image corresponding to one $H_{g}(\boldsymbol{r})$. Amplitude and phase terms will be calculated according to the equations

$$
\begin{aligned}
& I_{g}(\boldsymbol{r})=\Re\left(H_{g}(\boldsymbol{r})\right) \\
& P_{\boldsymbol{g}}(\boldsymbol{r})=\Im\left(H_{g}(\boldsymbol{r})\right)-\phi_{g}
\end{aligned},
$$


where $\Re$ and $\Im$ stands respectively for real and imaginary parts. The displacement field $\boldsymbol{u}(\boldsymbol{r})$, is a two-dimensional vector field, and to recover it in every direction it is necessary to reconstruct the $H_{g}$ of two non-collinear $\boldsymbol{g}_{1}$ and $\boldsymbol{g}_{2}$. Mathematically we need to find two vectors $\boldsymbol{a}_{1}$ and $\boldsymbol{a}_{2}$, in the real space, solving the equation

$$
\boldsymbol{u}(\boldsymbol{r})=-\frac{1}{2 \pi}\left[P_{\boldsymbol{g}_{1}} \boldsymbol{a}_{1}(\boldsymbol{r})+P_{\boldsymbol{g}_{2}} \boldsymbol{a}_{2}(\boldsymbol{r})\right]
$$

From the displacement field $\boldsymbol{u}(\boldsymbol{r})$ it is finally possible to calculate the strain tensor $\epsilon[8]$ :

$$
\epsilon=\left(\begin{array}{ll}
\varepsilon_{x x} & \varepsilon_{x y} \\
\varepsilon_{y x} & \varepsilon_{y y}
\end{array}\right)=\left(\begin{array}{ll}
\frac{\partial u_{x}}{\partial x} & \frac{\partial u_{x}}{\partial y} \\
\frac{\partial u_{y}}{\partial x} & \frac{\partial u_{y}}{\partial y}
\end{array}\right) .
$$

All these procedure are available as a series of script implementing numerically GPA reconstructions and strain calculations. The software gives the strain tensor as separated components expressed as follows [8]:

$$
\begin{array}{rlr}
\varepsilon_{x x}=\frac{\partial u_{x}}{\partial x} & \text { Symmetric strain } E_{x} \\
\varepsilon_{y y}=\frac{\partial u_{y}}{\partial y} & \text { Symmetric strain } E_{y} \\
\varepsilon_{x y}=\frac{1}{2}\left(\frac{\partial u_{x}}{\partial y}+\frac{\partial u_{y}}{\partial x}\right) & \text { Symmetric strain } E_{x y} . \\
\Delta_{x y}=\frac{1}{2}\left(\frac{\partial u_{x}}{\partial x}+\frac{\partial u_{y}}{\partial y}\right) & \text { Mean dilatation } D_{x y} \\
\omega_{x y}=\frac{1}{2}\left(\frac{\partial u_{y}}{\partial x}-\frac{\partial u_{x}}{\partial y}\right) & \text { Rotation } R_{x y}
\end{array}
$$

The definition of the $x$ and $y$ reference axis are chosen by the user at his own convenience.

GPA provides the instruments to reconstruct lattice deformations starting from the HREM image of a crystalline sample. All the limits of the technique are due to this specific image-based approach. As already stated, the image is representative of the lattice structure of the sample. Intensity features in the image, however, will be directly connected to the arrangement of planes in the sample only under restrictive conditions. A set of constraints is imposed by the HREM technique itself and others lie in the sample structure. It is beyond the scope of this manuscript to examine all the parameters determining the limits and the precision of the reconstructed strain maps [33]. Here it is important to note that the objective lens, and other imaging parameters, can strongly affect the result of the analysis. Most of the problems arising from the way the microscope transfers the spatial information from the specimen to the imaging plane can be minimized by using the latest generation aberration-corrected microscopes. The aberration-corrected microscope transfer function provides a faithful transfer of spatial frequency over a large range. The remaining geometric distortions induced by the lenses are removed by subtracting from the resulting phase images a reference deformation map specific to the microscope used. 
The sample itself can present some important problems in the analysis of the HREM images. Variations in the thickness of the sample in the area under investigation induce an additional geometric phase displacements that will be impossible to distinguish from those induced by changes in the interatomic distances. Strong intensity variations of the interference fringes and, in the limiting case, a contrast inversion, will be interpreted by the numerical routine as an additional phase displacement not related to any physical strain.

Graphene membranes resolve or minimize many of the above-mentioned problems. The most important limitation in TEM sample preparation is the control over the thickness of the specimen. In the case of FGC membranes the thickness is ideally uniform, and it can be experimentally determined at atomic level without error and is normally constant over a large area. In our analysis we will concentrate on the determination of the apparent compression induced by the vertical geometrical projection of the bent membrane. We thus have a quasi-perfect sample to investigate with the GPA technique.

\subsection{Experimental Reconstruction of Bent Graphene Membranes}

Particular care was taken in setting up the experimental conditions for HREM imaging. The experiments were performed using the aberration-corrected Tecnai F20 TEM available at the CNRS-CEMES of Toulouse (http://www.cemes.fr), operated at an acceleration voltage of $100 \mathrm{kV}$ to avoid structural damage to the carbon lattice. The sample was chosen according to the following criteria: it must have an explicit geometrical distortion, where the effect of projection induced apparent strain and real mechanical strain could be easily separated. Two kinds of sample were analyzed. The first were mechanically exfoliated graphite flakes, where thin electron transparent flakes can be easily obtained with thin borders composed of few-layers, typically folded over themselves. Natural graphite powder was exfoliated using a mortar and pestle and successively sonicated in isopropanol for additional exfoliation and dispersion. The resulting solution was drop-cast over standard $3 \mathrm{~mm}$ TEM holey carbon grids. The second kind of sample were graphene crystals grown by CVD on copper substrate and then transferred on a 3-mm TEM grid.

In Fig. 4 is shown the HREM image of a graphene flake prepared as highlighted before. The flake is folded over itself along two borders. From an analysis of the borders (0002) fringes it is possible to state that the flake is composed of three superimposed graphene layers (six total layers). By looking closer at the edges, as in inset (a) of Fig. 4, it is possible to determine the stacking order of the composing graphene sheets. The series of intensity peaks corresponding to the position of benzene rings in the stacked layers is highlighted by red circles. Their alignment along lines not perpendicular to the flake edge is characteristic of $\mathrm{ABAB}$ stacking. 


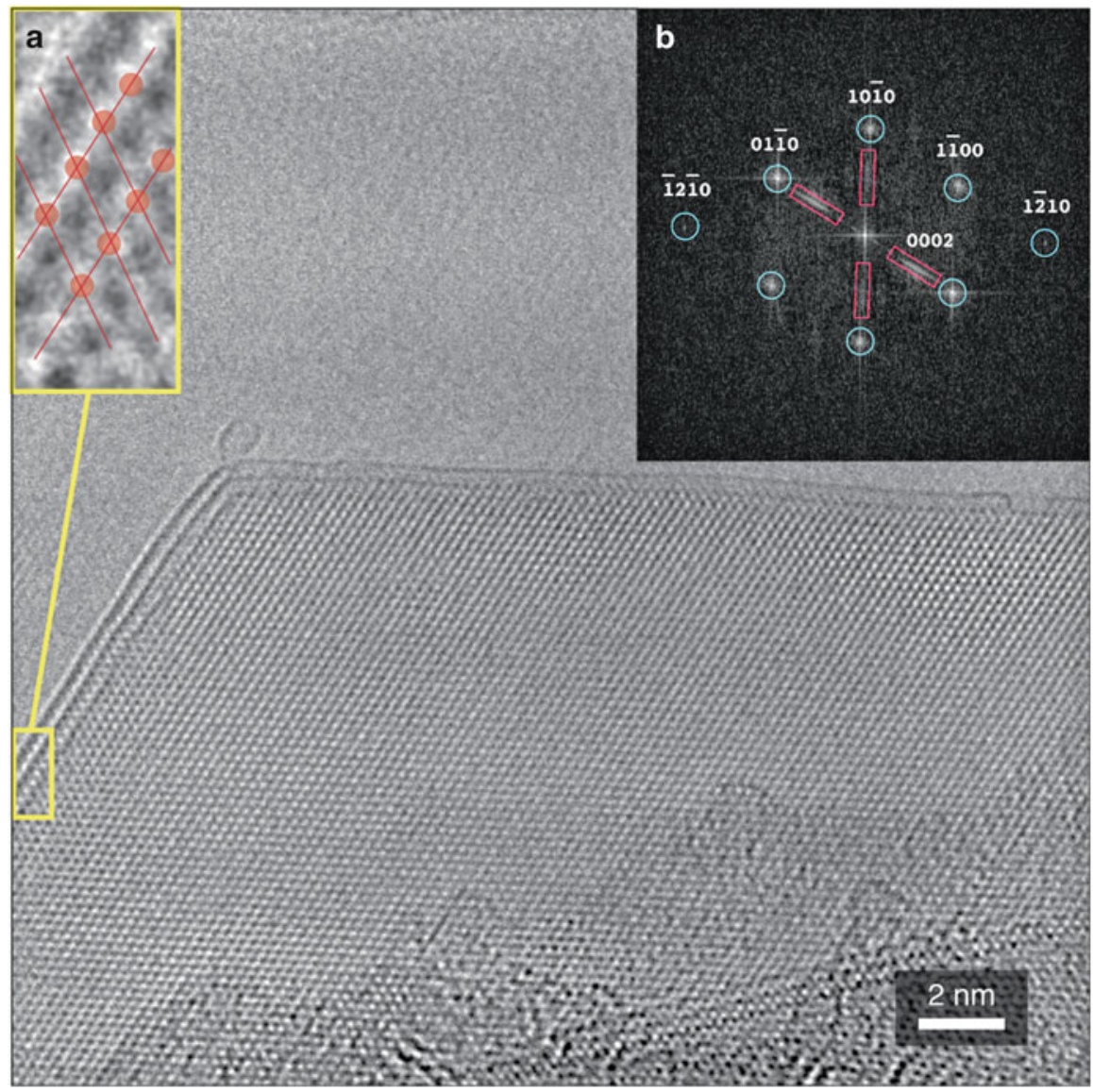

Fig. 4 HREM image of the border of an FGC flake. The membrane is folded over itself on two sides, exposing (0002) fringes, which makes possible to determine the number of layers in the membrane as 3 . The inset $(a)$ shows a close-up of the (0002) folded zone in the yellow rectangle. From the disposition of the intensity peaks it is possible do determine that the flake has $A B A$ staking sequence. The inset $(b)$ shows the FFT of the HREM image. Graphite reflections are marked for easier view (blue circles) and $(0,0,0,2)$ reflections of the two borders are clearly visible (red rectangles)

Inset (b) of the same figure shows the FFT spectrum of the image. Graphite principal reflections are highlighted (blue circles) along with folded borders (0002) reflections (red rectangles).

An important feature of the image is the defocus difference which is apparent between the left-side and upper borders. The left border shows some evident Fresnel fringes due to under-focus, while the upper border is almost at focus, with no Fresnel fringe visible. This suggest that there is some height difference between the two regions, leading to the hypothesis that the membrane bends near the border, inducing a compression of the projected atomic positions. 
Fig. 5 Proposed schematics for the structure of the folded flake under investigation

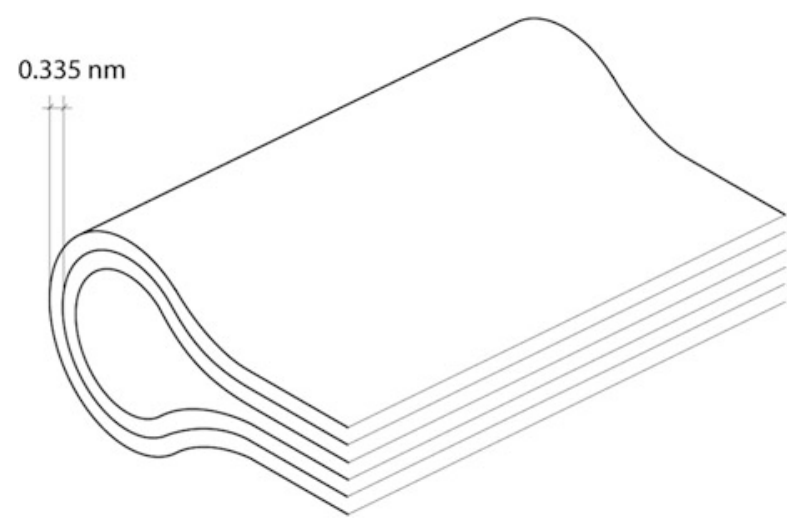

Following this hypothesis, it is possible to make a model of the 3D structure of the folded border of the graphene flake, as reported in Fig. 5. The three-layers flake starts to bend, makes a curve, and folds over itself forming six stacked layers.

Looking back to the experimental image, it is worth noting that this flake is folded along particular directions. Indeed, looking at the FFT spectrum of the image we note that the upper and left border are folded respectively perpendicularly to $(01 \overline{1} 0)$ and $(10 \overline{1} 0)$ lattice direction. This means that when the three layers superimpose after the border bending they will stack over the original three matching lattice positions and, eventually, preserving the overall ABAB stacking.

What is important to note is that in this case the bending, and therefore the apparent strain in the projected lattice image, will be only in one direction. The sample is therefore in a suitably simple configuration to test GPA 3D reconstruction. It is important to stress here that a hypothesis of 3D structure of the flake, as shown in the schematics of Fig. 5, can be made from basic knowledge of the material and from a careful inspection of the HREM image itself (geometry, defocus variations, etc.). However, it is not possible in any way to quantify the surface height variation from a standard analysis of the HREM image. We will show that this can be obtained by GPA.

The first step in GPA is the reconstruction of the phase displacement maps relative to at least two non collinear $\boldsymbol{g}$ vectors. In the case of the flake under investigation we selected $(01 \overline{1} 0),(10 \overline{1} 0)$, and $(1 \overline{1} 00)$ reflections of the reciprocal lattice. Figure 6 shows the results of the reconstruction. To select the $\tilde{H}_{g}(\boldsymbol{k})$ coefficients a numerical mask has been used, with an aperture corresponding to a final resolution of about $0.5 \mathrm{~nm}$ in the reconstructed phase maps.

The first noticeable feature of the phase maps of Fig. 6 is that large phase displacements can be seen in the (1010) direction, near the upper border. Figure 7 shows the phase map for the $(10 \overline{1} 0)$ direction. Three triangular regions of significant phase displacement are aligned over the border and are indicated by white arrows. The right one is the larger and the most intense. A slight phase displacement is noticeable corresponding to these regions in the $(1 \overline{100})$ direction, while in the $(01 \overline{1} 0)$ direction the phase is almost flat all over the flake. 


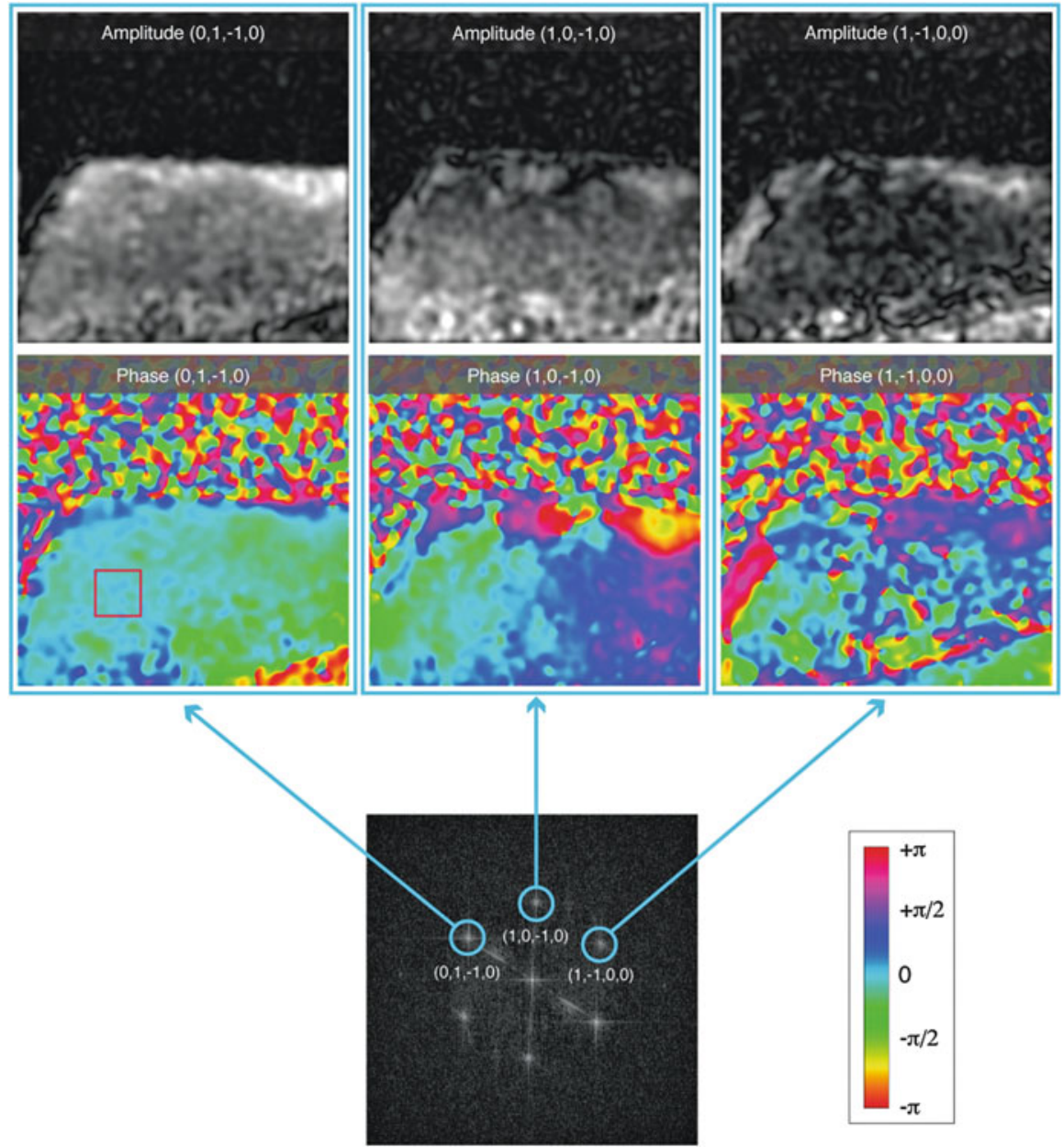

Fig. 6 Reconstructed amplitude and phase maps for the graphite reflections indicated by blue circles in the FFT of the image of Fig. 4. Variations in the phase values are mapped with a color scale. Large phase variations are visible near the upper border in the reconstructed phase map from the $(1,0, \overline{1}, 0) \boldsymbol{g}$ vector. Reference areas to re-normalize the phase backgrounds were taken in the regions corresponding to that marked by the red rectangle in the $(0,1, \overline{1}, 0)$ phase map. The lateral dimension of the reconstructed phase maps is identical to that of the original HREM image $(27.60 \mathrm{~nm})$

The apparent compression we are looking for is therefore acting displacing (10 $\overline{1} 0)$ and $(1 \overline{100})$ fringes, leaving almost unmodified the $(01 \overline{1} 0)$ direction. To calculate strain maps we need to define a reference axis to project their components. A possible choice is the direction $(2 \overline{1} \overline{1} 0)$, assuming the flake is bent with a slope in that direction. 
Fig. 7 Phase map reconstructed for the $(10 \overline{1} 0)$ direction. Arrows indicate large phase variations at the border of the flake

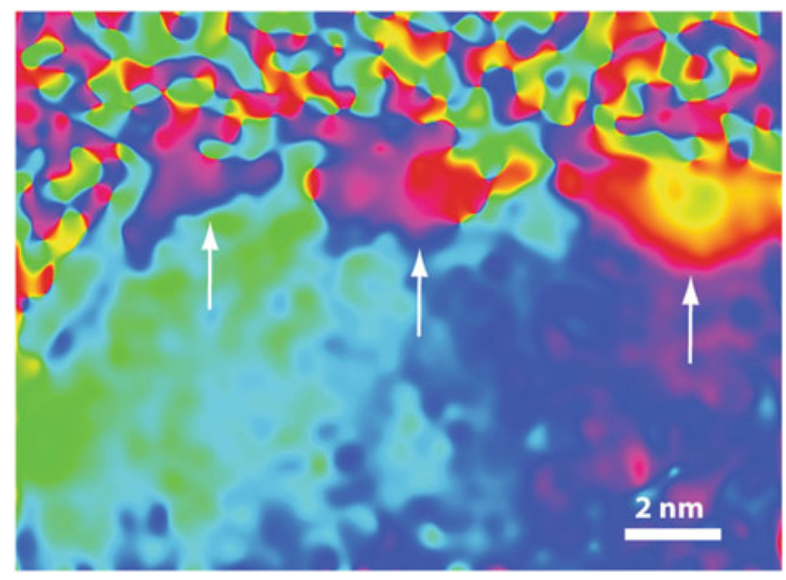

Figure 8 shows a close-up of the border region in HREM imaging and in the (1010) phase map. The plot of Fig. $8 \mathrm{c}$ is taken in the marked region and shows that a large phase variation is local along the direction of the $(2 \overline{1} \overline{1} 0)$ lattice planes. We can form a hypothesis for the model of the atomic structure of the flake in this region as shown in Fig. 8d. According to the scheme in Fig. 5, three layers bend in the positive $z$ direction while the other three bend in the opposite direction, creating a hollow space near the border curvature.

The calculated amplitude maps are generally slowly varying, except for some localized regions for $(1 \overline{100})$ and $(01 \overline{1} 0)$. Between the large phase bumps of the (1100) map, localized contrast inversions will be likely to generate artifacts during strain calculations [33]. The same problem is present in the marked region of the (0110) amplitude map, near the left border. We will avoid these regions during analysis of the calculated strain maps.

This structural hypothesis will be verified by calculating the strain field map when choosing the Cartesian reference system indicated in Fig. 8a, i.e., with the $x$-axis in the $(2 \overline{1} 10)$ direction. As already discussed, we need two phase maps calculated for two non-collinear directions to recover the 2D strain field. Every couple of $\boldsymbol{g}$ vectors is mathematically equivalent, so a good criterion will be to choose the couple resulting in the higher signal-to-noise ratio. We checked different combinations and all the results were consistent. In the end, the best results have been obtained using the $(01 \overline{1} 0)$ and $(10 \overline{1} 0)$ directions.

Figure 9 shows the results for the strain maps $\varepsilon_{x x}$ and $\varepsilon_{y y}$. They are significantly more affected by noise than the phase images because of the numerical process of calculating the derivative of the phase [34]. Nevertheless, the main features of the maps can be easily identified.

The central part of the flake is almost strain free in both the $x$ and $y$ components. Along the $x$-direction we recognize some strain change associated with the borders of the three regions already noted near the upper border. Consistent with the choice of axes, the largest strain variation is associated with the zone highlighted in Fig. 8a, b). 

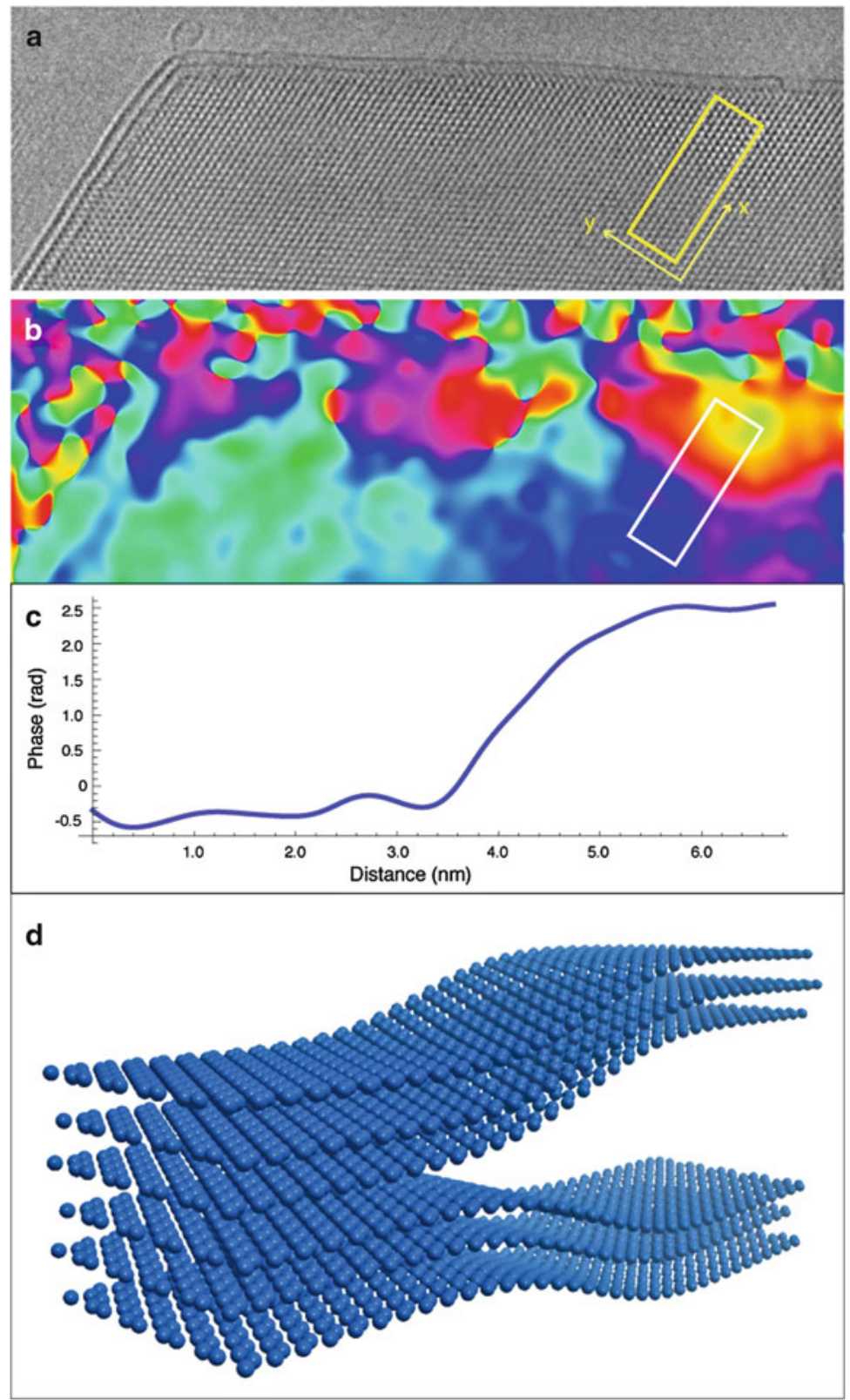

Fig. 8 (a) HREM image of the flake near the upper border. The region of interest (ROI) is marked by the rectangle and the Cartesian reference system for further analysis is indicated. (b) Corresponding region of the $(01 \overline{1} 0)$ phase map. The same ROI of (a) is reported. (c) Plot of the phase profile along the $x$-axis in the region of interest. (d) Schematics of the atomic structure of the flake in the ROI 

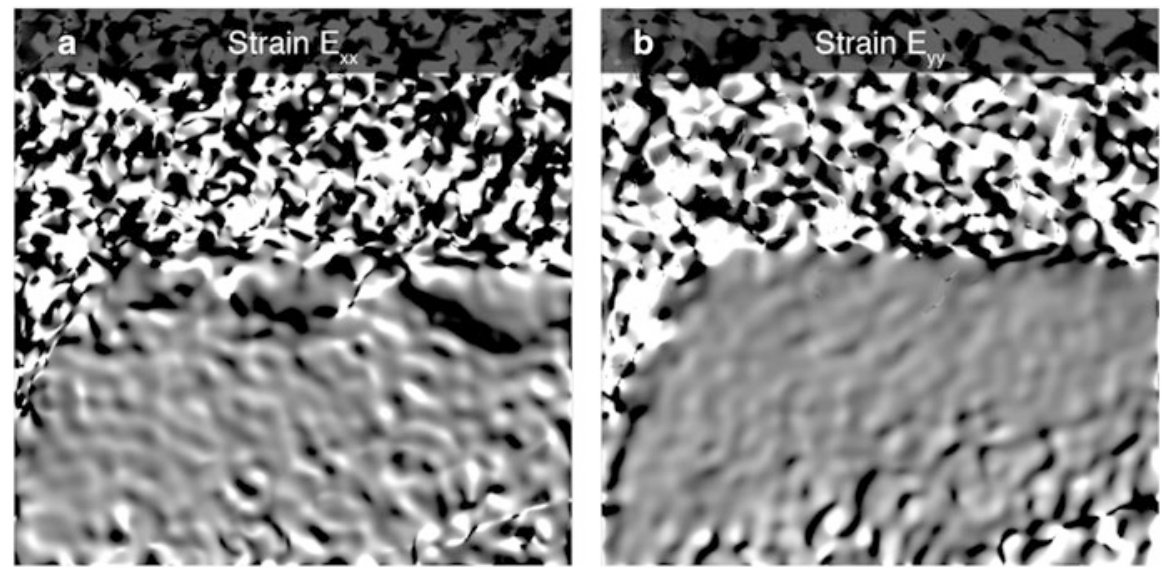

Fig. 9 Calculated strain field maps. (a) Components of the strain field along the ( $2 \overline{1} \overline{1} 0) x$-direction and (b) along the $(01 \overline{1} 0)$ perpendicular $y$-direction
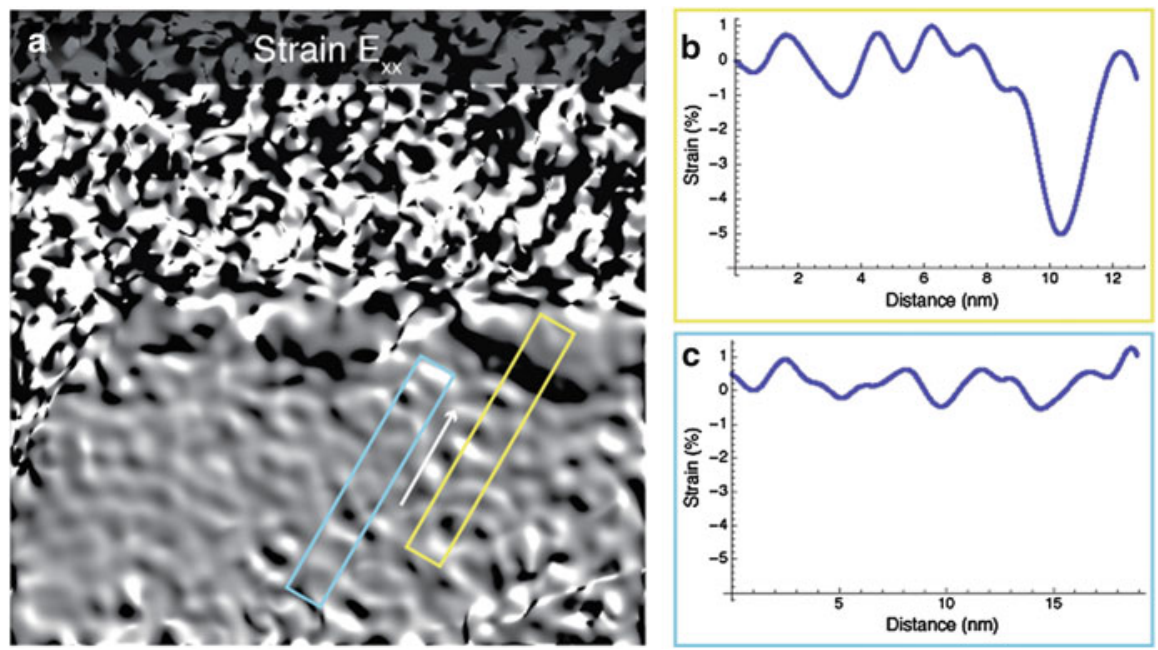

Fig. 10 (a) Calculated strain map $E_{x x}$ in the (21̄10) direction. (b) Profile of the strain intensity along the yellow ROI. (c) Profile of the strain intensity for the blue ROI. Profiles are acquired in the direction indicated by the white arrow

To recover the local deformation of the membranes from the strain maps we should retrieve the local slope value from the measured strain. For this purpose we need to find a zone were the apparent compression can be assumed to be along one direction only.

Recalling Fig. 8, it is possible to identify a region showing local uniaxial strain due to the geometric projection of the bent 3D atomic structure. The zone corresponds to the region marked with the yellow rectangle in Fig. 10a. According 
to the one-dimensional model previously discussed, we should find the peak of the compression associated to the position of the curvature inversion point in the bent flake.

Figure $10 \mathrm{~b}$ shows the profile of the strain along the yellow rectangle. The plot indeed shows a localized peak in the strain corresponding to the middle region of the phase ramp of Fig. 8c. The measured compression is about $5 \%$. Such a value is extremely high for a pure mechanical in-plane compression of the crystal lattice.

Taking into account that graphene has a Young's modulus of about 1 Tpa, a 5\% compression implies a stress of about $10^{28} \mathrm{~N} / \mathrm{nm}^{2}$. Even if graphene should resist such a force without breaking the interatomic bonds, a compression like this would result in a $3 \mathrm{D}$ deformation. Thus, once more, we consider that such a strain is apparent, and due to the effect of projection of the bent flake on the $x y$-plane.

The profile of the unstrained central area (blue rectangle of Fig. 10c) enables analysis of the noise of the image. The two plots of Fig. 10 are obtained using the same intensity scale to visualize background oscillations easily. An average of the strain free area background shows noise oscillations of about $0.6 \%$. Such oscillations are mainly due to the poor contrast of the graphite fringes in the HREM image. This noise is amplified by the numerical calculations performed by the GPA to obtain the derivative maps, and it is the first limitation of this technique. Nevertheless, in this case, a relatively good signal-to-noise ratio, with a few percent strain, is still present.

For each value of the position of the map, a value for the slope of the surface of the flake can be calculated, by means of simple trigonometry, that it is able to define immediately the local angle $\alpha$ between the flake surface and the $x y$-plane in terms of the strain $\varepsilon$ as

$$
\cos (\alpha)=(1-\varepsilon)
$$

From the value of $\alpha$ it is straightforward to calculate its tangent for each value of the strain. Then, the reconstruction of the 3D atomic structure of the graphite flake can be calculated from the fit of the local slope, which is the first derivative of the height displacement of the flake surface. A straight integration can give directly the atomic positions.

Figure 11 shows the results of the structure simulation. In the inset of Fig. 11a the integrated height as a function of the distance is plotted, and the three dimensional model is calculated accordingly. Each stacked layer change its $z$ position of about $0.8 \mathrm{~nm}$, as indicated in the lateral projection of Fig. 11b. It is almost certain that the bent structure of the three-layers graphene flakes can be reconstructed only from the analysis of the apparent strain in the HREM image.

The same method can be applied to a single graphene layer as that reported in Fig. 12 [35]. The membrane was grown by chemical vapor deposition (CVD) on a copper substrate and transferred on a TEM grid. The HREM image shows the folded edge of a monolayer membrane; the border is visible by the (0002) graphite lattice fringe and the two layers in the stacked regions are rotated by an angle $\theta=21.7^{\circ}$, as shown by the fast Fourier transform (FFT) in the bottom left corner inset. 
Folds and Buckles at the Nanoscale: Experimental and Theoretical...

a

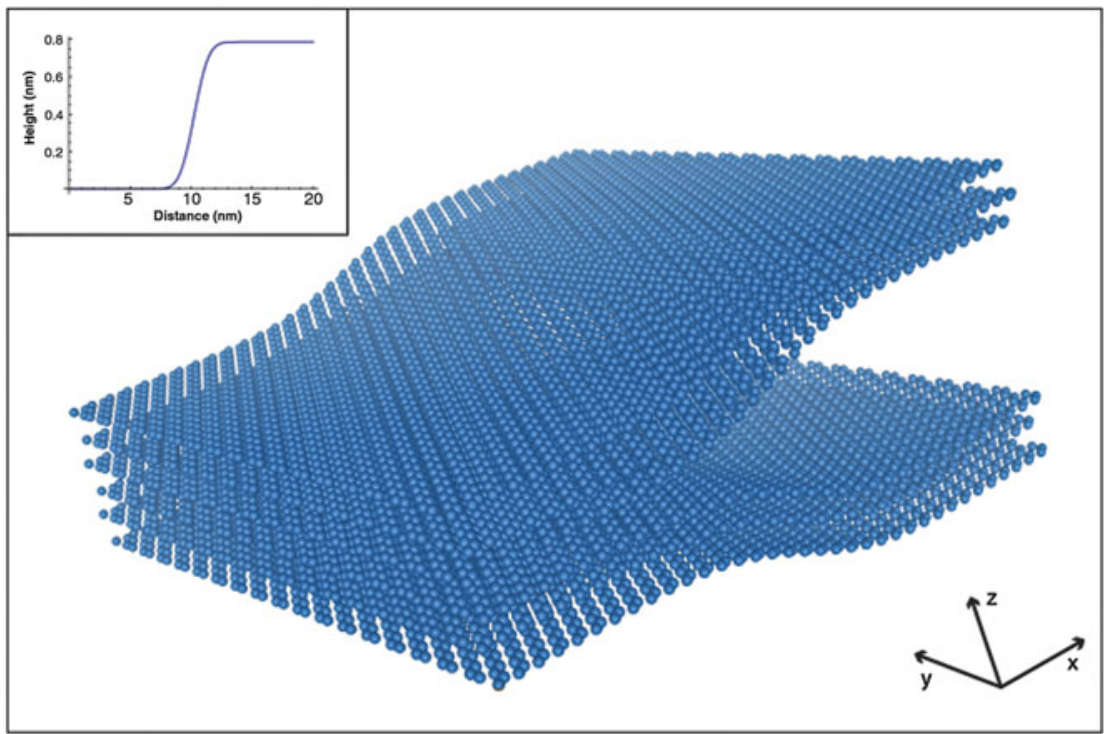

b

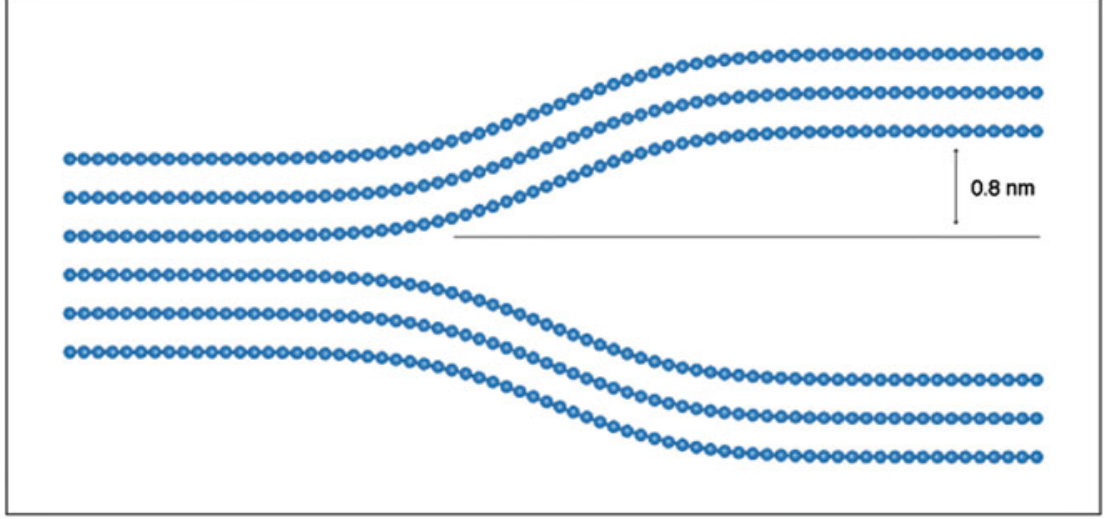

Fig. 11 Schematics of the reconstructed structure of the flake. (a) Perspective view. In the inset a plot of the elevation of each graphene layer. (b) Lateral view of the structure

In the top right corner a schematics of the superimposed lattices as they appear projected in the image is given. In addition it is clearly shown that the membranes are not atomically clean and adsorbates or residues from the growth are either on top or in between the membranes, changing its three dimensional configuration.

Figure 13 reports the resulting strain map in the direction perpendicular to the folded border. In the map a compression running along the border is clearly visible, close to a relaxed central area, where the two lattices are in contact and a strained internal region, parallel to the border. Analyzing the deformation in the two compressed region along the border, marked with 1 and 2 (see the profiles on the right of Fig. 13), and following the same procedure described before, it is possible 


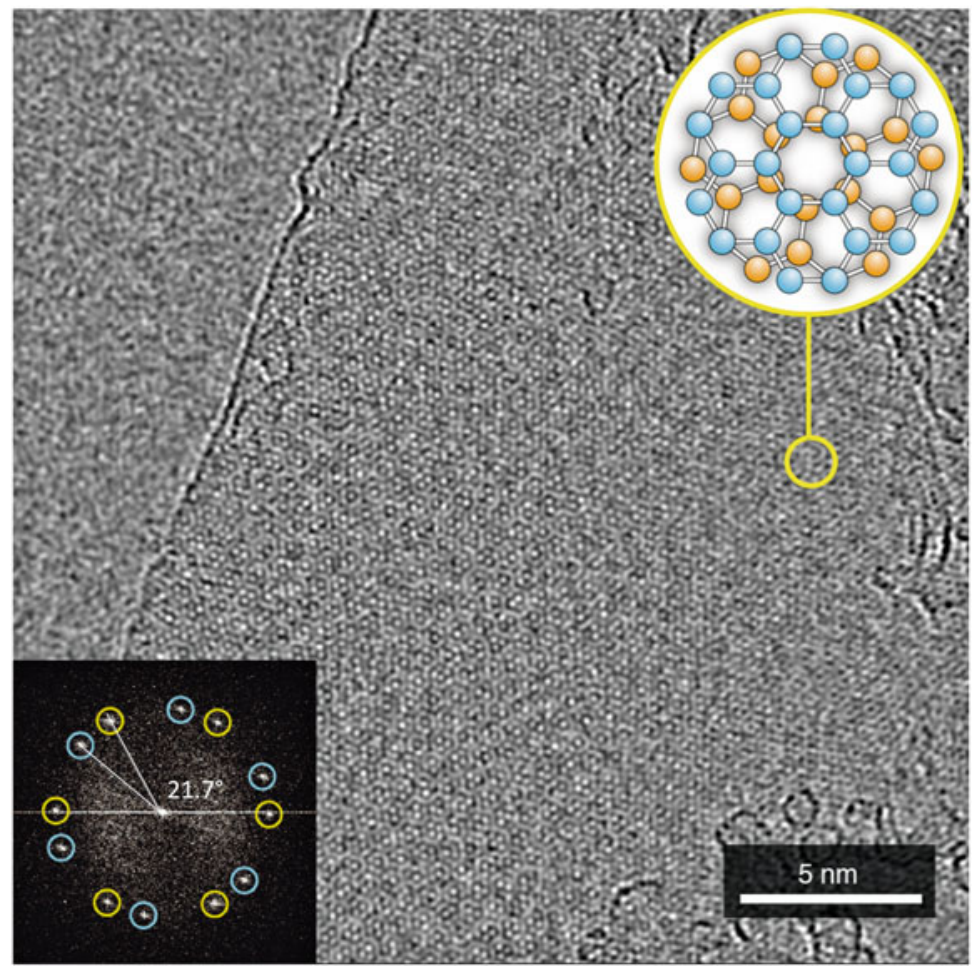

Fig. 12 HREM image of a single layer graphene flake folded edge. Bottom left corner: FFT of the image, showing the stacking orientation of the two lattices. Top right corner: schematics of the folded lattice

to interpret the strain as apparent and induced by the curvature of the fold, and therefore to estimate a maximum slope of $16^{\circ}$ over a length of $3 \mathrm{~nm}$ in both regions, corresponding to a height variation of $0.8 \mathrm{~nm}$.

Another example of a graphene flake showing a more pronounced 3D structure is reported in Fig. 14, where a different region of the same border of the flake shown in Fig. 12 is reported. The HREM image in Fig. 14a clearly shows an isolated defect on the folded border, indicated by the white circle, and two lines of compression joining at the defect site at the border, highlighted with the white lines. These curved compressed regions are clearly shown in the strain map in Fig. 14b. As before, we can analyze the strain along the two regions marked with (1) and (2), therefore measuring a height variation of about $1 \mathrm{~nm}$ over a length of $4 \mathrm{~nm}$ with a slope of $16^{\circ}$ in region 1 , and of $0.9 \mathrm{~nm}$ over a length of $2 \mathrm{~nm}$ and a slope of $27^{\circ}$ in region 2 . Therefore we can interpret the observed compression lines as a curved wrinkle induced in the folded edge by the defect, and the two membranes are wrapping in their interior the carbonaceous contaminants shown in the HREM micrograph.

It is worth noting that in all the reconstructed structures, close to the fold the curvature of the membrane is expected to increase up to $90^{\circ}$, and this is not 
Folds and Buckles at the Nanoscale: Experimental and Theoretical...
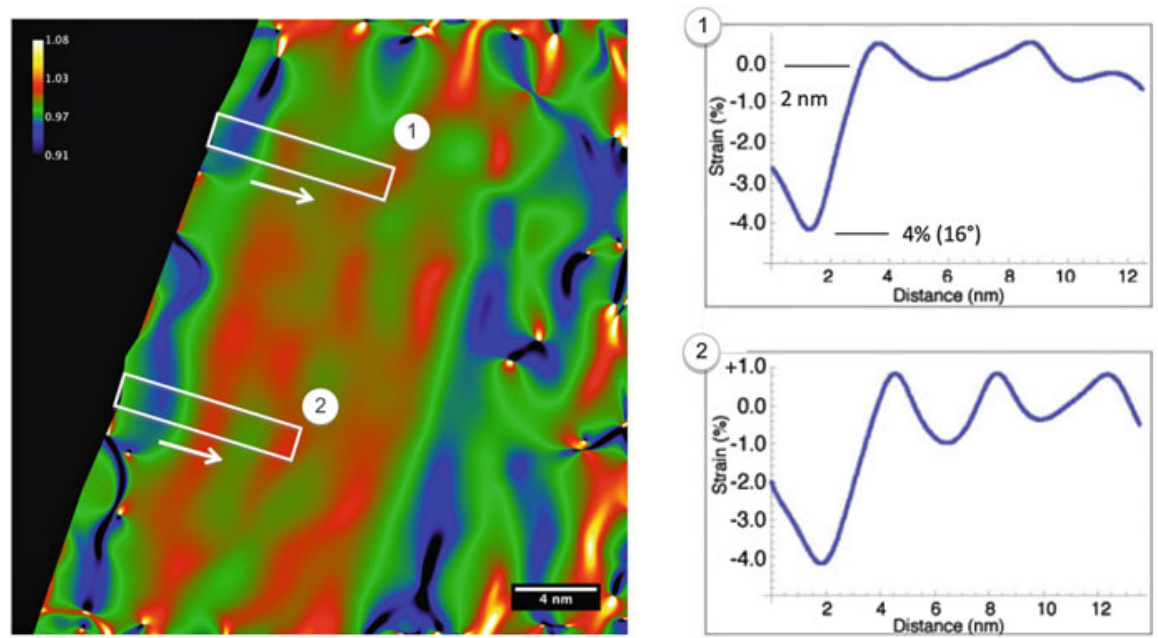

Fig. 13 Map of the strain component in the direction perpendicular to the edge of the fold. The internal part of the flake shows no significant strain, while parallel to the border we can observe compressed regions. $(1,2)$. On the right are reported the strain profiles acquired, respectively, over regions (1) and (2)
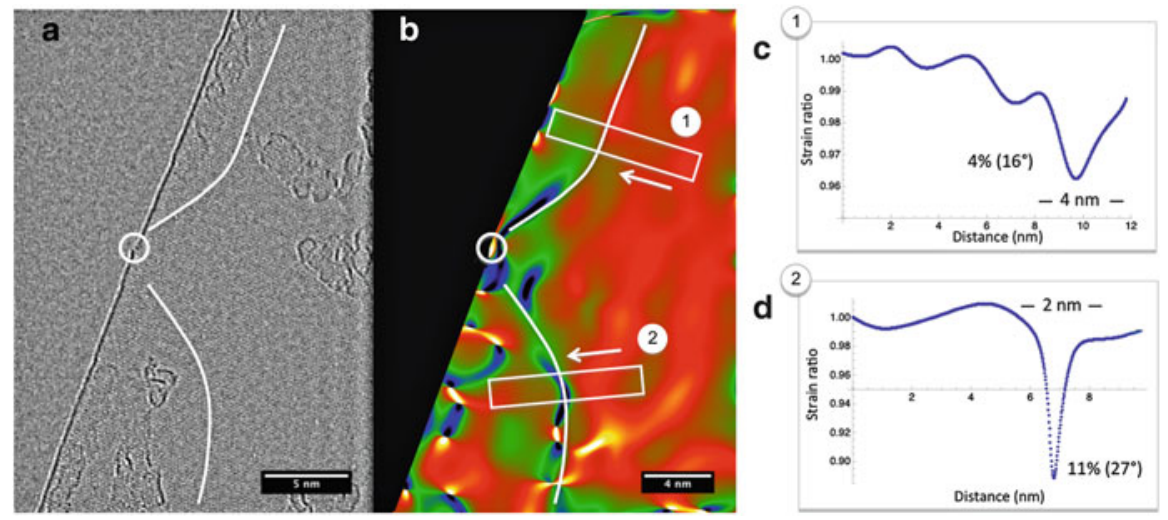

Fig. 14 (a) HREM image of a single graphene layer folded over itself. A point defect, highlighted by the white circle, is visible on the border; (b) map of the strain component in the direction perpendicular to the edge of the fold. Two compressed lines, highlighted with white lines in (a), are clearly visible; (c, d) strain profiles acquired, respectively, over regions (1) and (2)

highlighted in the apparent strain maps. In fact, this fold corresponds to an infinite apparent compression in the imaged lattice, and in the strain profiles it is hidden by geometric phase artifacts arising from the phase discontinuity at the interface between the flake and the vacuum. In addition, the spatial resolution achieved in the GPA reconstruction is $0.5 \mathrm{~nm}$, which is the same order of the estimated fold curvature radius, making it impossible to map such a large and rapid variation of the crystal slope. 
Nevertheless, in all the reported cases the method demonstrated effective in the reconstruction of the 3D structure of the graphene membranes, at least far from the region close to the folded edge. To validate the proposed methodology, the further step is to compare the experimental results with a model of the 3D structure of graphene flakes. This will be the subject of the next section.

\section{Modeling the Bending Properties of Graphene Membranes: Conceptual Framework}

In order to obtain trustworthy HRTEM simulated images addressed at validating the experimental results, the actual 3D atomic structure of folded graphene membranes is needed. With this aim, we proceed through a multi-step protocol, obtained by blending together atomistic and continuum modeling:

- The shape of a folded two-dimensional continuum membrane is at first predicted according to continuum elasticity.

- The shape corresponding to the minimum elastic energy configuration is then decorated by a carbon honeycomb lattice.

- Careful lattice relaxation follows, eventually driving to the actual atomistic configuration of a folded graphene sample.

A key-feature underlying the above protocol is that, while the bending processes of a two-dimensional continuum membrane involves only out-of-plane deformations, in a two-dimensional atomic lattice such a deformation pattern cannot be achieved without introducing bond strain [18]. This is mainly due to the distortion and mutual interaction between neighboring $p_{z}$ orbitals. Thus, there is always interplay between real bond-length variations and the apparent strain observed by projection of a bent structure onto a plane. Taking into account properly the bond strain induced by bending is, in turn, a tough problem owing to the peculiar nature of the carbon-carbon interactions which can only be quantitatively modeled by quantum mechanics. Therefore, the atomistic relaxation outlined above is performed by tight-binding molecular dynamics, making use of the representation by Xu et al. [36].

\section{Simulation Protocol}

\subsection{Step 1: Predicting the Shape of a Folded Continuum Membrane}

From the continuum elasticity theory point of view, the equilibrium shape of a folded graphene (also known as BLE, Bilayered Edged Graphene) is the same as that of any other $2 \mathrm{D}$ solid membrane and, hence, can be predicted by solving 
a

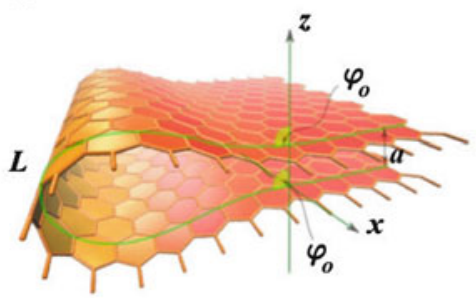

b

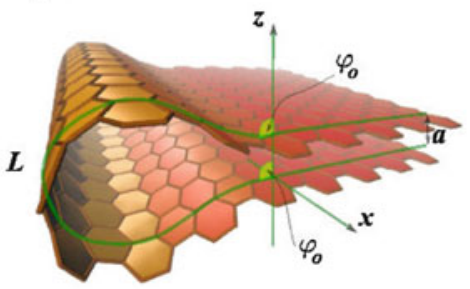

Fig. 15 3D rendering of the TB simulated graphene folds. (a) Armchair fold. (b) Zig-zag fold

the Euler-Poisson problem, just providing the correct geometrical boundary conditions.

The geometrical features of the specific configuration of a BLE graphene is achievable by imposing the correct length $(L)$ of the bent carbon ribbon (i.e., as shown in Fig. 15, one can recognize two different regions, the inner one that is almost a flat bilayer of graphene and the bent closed edges), the attack angle $\left(\varphi_{o}\right)$ (i.e., the angle obtained between the tangent plane at the folded region and the flat one where they match together), and the (almost) constant distance ( $a$ ) between the two parallel layers along the flat region (see Fig. 15).

The length of the folded region in graphene results from the competition between the bending momentum (i.e., the bending moduli of graphene, the bending rigidity, and the Gaussian bending stiffness, depending on the mean and Gaussian curvatures, respectively) and the attractive Van der Waals potential, which engender the opening and the adhesion of the graphene sheet, respectively. In the matter of the geometric features, the generic cylindrical configuration observed in BLE involves only the mean curvature on the surface, and therefore the elastic energy stored by the curvature at certain conditions depends just on the bending rigidity (see Sect. 4.2).

The bending energy density $\mathcal{U}_{b}$ of a generic surface can be written as $\mathcal{U}_{b}=$ $2 \kappa \mathcal{H}^{2}-\bar{\kappa} \mathcal{K}$, where the mean curvature is $\mathcal{H}=\frac{1}{2}\left(k_{1}+k_{2}\right)$ and the Gaussian curvature is defined as $\mathcal{K}=k_{1} k_{2}, k_{1}=R_{1}^{-1}$ and $k_{2}=R_{2}^{-1}$ are the principal curvatures, while $R_{1}$ and $R_{2}$ are the local principal radii of curvature. Due to the Gaussian curvature being null, $\mathcal{K}=0$, in the case of cylindrical geometry, the bending energy density $\mathcal{U}_{b}$ is given by $\mathcal{U}_{b}=\frac{1}{2} \kappa k_{1}^{2}$.

The corresponding problem consists in finding the curve $z=z(x)$ by minimizing the bending energy $U_{b}=\iint \mathcal{U}_{b} d \sigma$ under the given boundary conditions that consist in fixing the positions of the two parallel edges (with length $l$ ) at a given distance $a$ (i.e., the equilibrium distance of a bilayered graphene, $a=1.41 \AA$ ), a constrained width $L$ (i.e., enforcing the absence of any in-plane stretching), and the attack angles are fixed for continuity reasons by the bilayer flat region. 
Fig. 16 A cross-section of a bent ribbon (blue curve) with parallel edges at fixed distance $a=3.4 \AA$, i.e., the equilibrium distance between two graphene layers. The ribbon width $L$ and the edges distance $a$ are taken as constant, and the attack angles $\theta_{o}$ and $\theta_{o}^{\prime}=-\theta_{o}$ (or $\phi$ and $\phi^{\prime}$ ) are fixed at $\pi / 2$

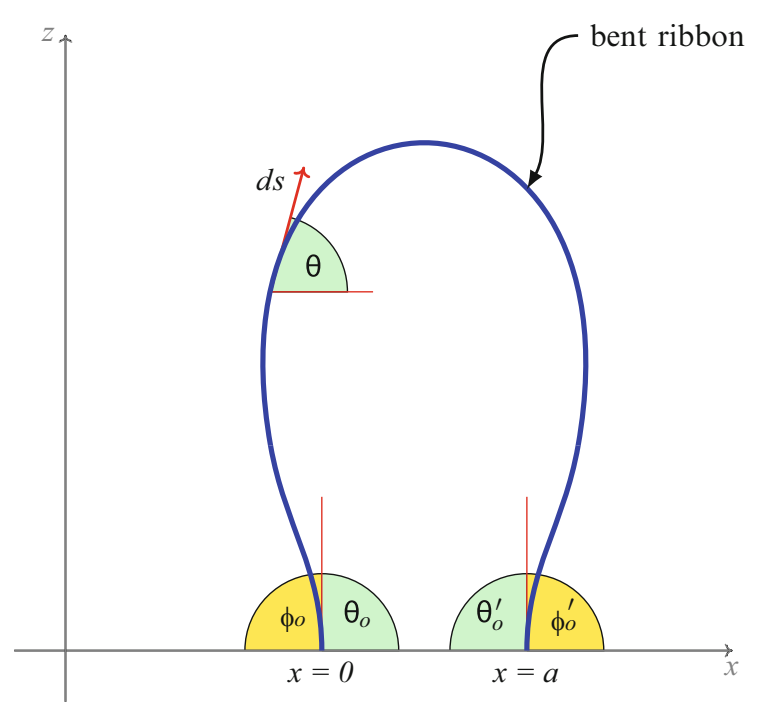

In Fig. 16 the cross section of the bent graphene ribbon is sketched as a line with width $L$. Hence, by imposing the method of Lagrange multipliers:

$$
U_{b}=\frac{1}{2} \kappa l \int_{0}^{a} \mathrm{~d} x\left(\frac{\ddot{z}^{2}}{\left(1+\dot{z}^{2}\right)^{\frac{5}{2}}}+\lambda \sqrt{1+\dot{z}^{2}}\right) .
$$

The above integral can be written in the general form $G(z)=\int_{0}^{a} \mathrm{~d} x \mathcal{F}(z, \dot{z}, \ddot{z}, x)$, which is the solution of the Euler-Poisson differential equation $\frac{\partial \mathcal{F}}{\partial z}-\frac{d}{d x} \frac{\partial \mathcal{F}}{\partial \dot{z}}+\frac{d^{2}}{d x^{2}} \frac{\partial \mathcal{F}}{\partial \ddot{z}}=0$.

By the application of constrained variational calculus we eventually obtain the final geometry in parametric representation $[x(s), z(s)]$ with the given boundary conditions. First of all, by the angle definition we get $\dot{z}=\tan \theta$, and $\ddot{z}=\frac{1}{\cos ^{2} \theta} \frac{\partial \theta}{\partial x}$. Introducing the arc length $s=\int_{0}^{x} \mathrm{~d} x \sqrt{1+\dot{z}^{2}}$, the Euler-Poisson differential equation can be written as

$$
\left(\frac{d \theta}{\mathrm{d} s}\right)^{2}=+\lambda+C_{1} \sin \theta+C_{2} \cos \theta
$$

By imposing the fixed attack angle condition, i.e., $\theta(0)=\theta_{o}=\pi / 2$ and $\theta(L)=-\theta_{o}$, as shown in Fig. 16, (14) leads to $C_{1}=0$, and can be simplified as $\frac{d \theta}{\mathrm{d} s}=-\sqrt{\lambda+C \cos \theta}$, where $\boldsymbol{C} \equiv \boldsymbol{C}_{2}$ as well as in the following. 
Finally, by using the length $L=\int_{0}^{a} \mathrm{~d} x \sqrt{1+\dot{z}^{2}}$ and the distance $a$ to obtain the parameters $\boldsymbol{C}$ and $\lambda$ it follows that

$$
\begin{aligned}
& L=\int_{-\theta_{o}}^{\theta_{o}} \frac{d \theta}{\sqrt{\lambda+C \cos \theta}} . \\
& a=\int_{-\theta_{o}}^{\theta_{o}} \frac{\cos \theta d \theta}{\sqrt{\lambda+C \cos \theta}} .
\end{aligned}
$$

Turning back in Cartesian coordinates, ${ }^{1}$ we have obtained the parametric form of the minimized surface:

$$
\begin{array}{r}
x=L \frac{\int_{\theta(s)}^{\theta_{o}} \frac{\cos \theta d \theta}{\sqrt{(\lambda+C \cos \theta)}}}{\int_{-\theta_{o}}^{\theta_{o}} \frac{d \theta}{\sqrt{(\lambda+C \cos \theta)}}} \\
z=L \frac{\int_{\theta(s)}^{\theta_{o}} \frac{\sin \theta d \theta}{\sqrt{(\lambda+C \cos \theta)}}}{\int_{-\theta_{o}}^{\theta_{o}} \frac{d \theta}{\sqrt{(\lambda+C \cos \theta)}}} .
\end{array}
$$

\subsection{The Bending Rigidity of Graphene}

The bending rigidity of graphene $(\kappa=1.40 \mathrm{eV})$, including relaxation effects, can be evaluated using carbon nanotubes instead of nanoribbons. Nanotubes, of course, do not show any edge effects, but the bending rigidity depends on the mean curvature, which in nanotubes is a geometric constant (the cylindrical geometry of a nanotube imposes the Gaussian curvature null, $\mathcal{K}=0$ ). Including relaxation effects in the function of the nanotube radius $R$ it possible to extract the pure bending energy term by comparing the radius variation between the reference starting tube, which has all bonds equal to the perfect graphene, namely $1.41 \AA$, and the fully relaxed one. In fact, bond stretching is observed down to $(15,0)$ nanotubes [37-39]

The elastic energy density $\mathcal{U}\left[\mathrm{eVA}^{-2}\right]$ of a nanotube can be written as the sum of the strain energy density and the bending energy density:

$$
\mathcal{U}=\mathcal{U}_{s}+\mathcal{U}_{b}
$$

\footnotetext{
${ }^{1}$ We observe that $\frac{\mathrm{d} x}{\mathrm{~d} s}=\cos \theta$, and $\frac{\mathrm{d} z}{\mathrm{~d} s}=\frac{\mathrm{d} z}{\mathrm{~d} x} \frac{\mathrm{d} x}{\mathrm{~d} s}=\sin \theta$.
} 
The bending energy density $\mathcal{U}_{b}$ of a general given surface can be written as

$$
\mathcal{U}_{b}=2 \kappa \mathcal{H}^{2}-\bar{\kappa} \mathcal{K}
$$

where the mean curvature is $\mathcal{H}=\frac{1}{2}\left(k_{1}+k_{2}\right)$ and the Gaussian curvature is defined as $\mathcal{K}=k_{1} k_{2}, k_{1}=R_{1}^{-1}$ and $k_{2}=R_{2}^{-1}$ are the principal curvatures, while $R_{1}$ and $R_{2}$ are the local principal radii of curvature. Choosing a cylindrical configuration that involves only one curvature (i.e., $k_{1}=R_{1}^{-1}$ and $k_{2}=0$ ), the mean curvature is $\mathcal{H}=\frac{1}{2} k_{1}$, while the Gaussian curvature is null, $\mathcal{K}=0$.

Thus in the case of cylindrical geometry, the bending energy density $\mathcal{U}_{b}$ is given by

$$
\mathcal{U}_{b}=\frac{1}{2} \kappa k_{1}^{2} \text {. }
$$

The total bending energy $U_{b}$ can be calculated by performing the integral of the bending energy density $\mathcal{U}_{b}$ on the reference surface $\Sigma_{o}$, i.e., $U_{b}=\iint_{\Sigma_{o}} \mathcal{U} d \sigma=$ $1 / 2 \kappa l \int_{\gamma_{o}} k_{1}^{2} \mathrm{~d} s$, where $\Sigma_{o}=L_{o} l$ is the total area of the reference system, $\gamma_{o}=2 \pi R_{o}$ is the circumference of the cylinder with radius $R_{o}$, and $s$ is the arc length $\left(0<s<L_{o}\right)$. Note that the reference surface $\Sigma_{o}$ is defined as the surface of the corresponding rectangular flat slice which has been rolled to build the nanotubes, i.e., the unstrained graphene nanoribbon wherein all the bond length are equal to the equilibrium distance $d_{\mathrm{C}-\mathrm{C}}=1.41 \AA$ between a pair of neighbor carbon atoms.

The solution of the integral is as follows:

$$
U_{b}=\frac{1}{2} \kappa l \frac{2 \pi R_{o}}{R^{2}} .
$$

If the bending does not involve stretching, the radius $R$ after the relaxation of the nanotube has to be equal to the reference cylinder radius $R_{o}$. Therefore the bending energy can be simplified as

$$
U_{b}=\lim _{R \rightarrow R_{o}} \frac{1}{2} \kappa l \frac{2 \pi R_{o}}{R^{2}}=\frac{\pi \kappa l}{R_{o}} .
$$

Because the bending energy can be computed by atomistic simulation as the difference between the total energy of the nanotube $E_{o}^{\text {tube }}$ and the corresponding reference flat system $E_{o}^{\text {flat }}$, namely $U_{b}=E_{o}^{\text {tube }}-E_{o}^{\text {flat }}$, the bending rigidity $\kappa$ of a nanotube with radius $R_{o}$ is given by

$$
\kappa=\frac{R_{o} U_{b}}{\pi l},
$$

in the absence of stretching on the surface (Fig. 17). 
Folds and Buckles at the Nanoscale: Experimental and Theoretical...

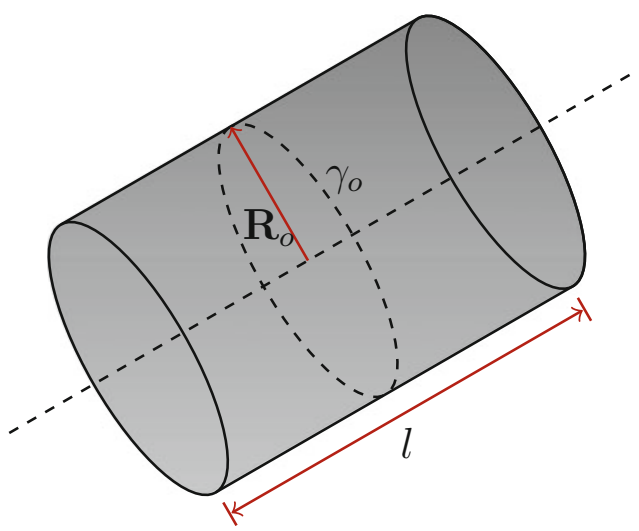

Fig. 17 A nanotube can be sketched as a simple cylinder. Here the radius $R_{o}$ and circumference $\gamma_{o}$ are referred to the reference configuration (i.e., without bond stretching), while the length $l$ is fixed by imposing the periodic boundary condition along the cylinder axis (dashed line)

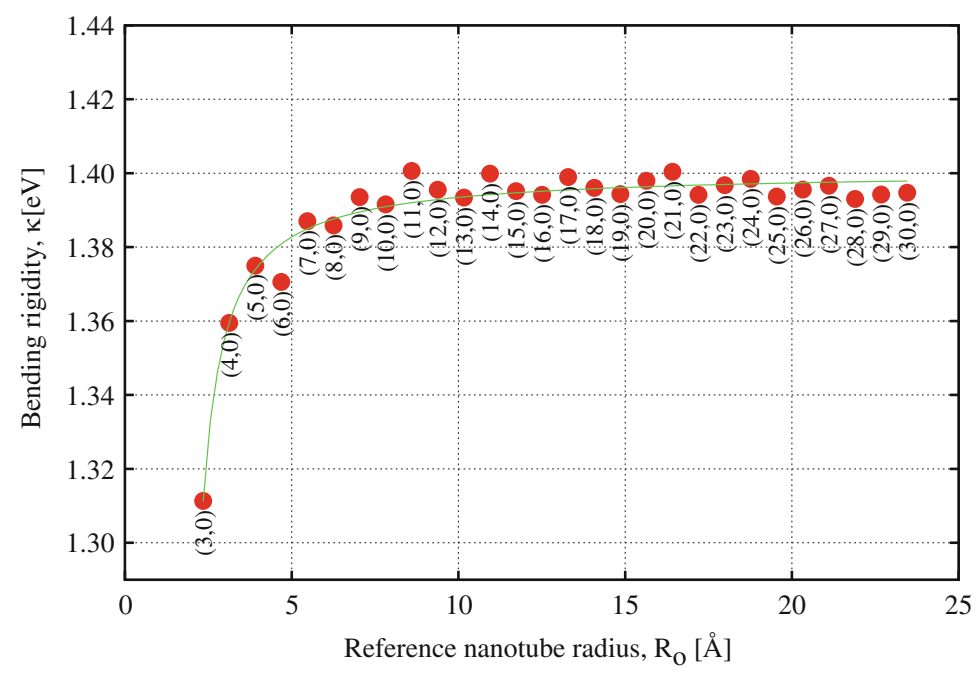

Fig. 18 Bending rigidity $\kappa$ in function of the radius of a set of zig-zag nanotubes in the range of $(3,0)-(30,0)$. The symbols show the value of the bending rigidity, as defined in (20), obtained by tight-binding simulations. Note that down to $(15,0)$ a deviation from the constant value is observed. This fact is due to the rising of stretching bond effects due to the curvature. The asymptotic value is $\kappa=1.40 \mathrm{eV}$

However, if the nanotube dimension is down to a certain radius (see Fig. 18), the relaxation of the structure allows a variation of radius $R$. In these cases with $R \neq R_{o}$, it needs to take in account the non-negligible stretching term in (16). Thus, the stretching energy density $\mathcal{U}_{s}$ has to be integrated as follows: 


$$
U_{s}=\frac{1}{2} \frac{E}{1+\nu} \iint_{\Sigma_{o}}\left(\operatorname{Tr}\left(\hat{\varepsilon}^{2}\right)+\frac{\nu}{1-\nu}[\operatorname{Tr}(\hat{\varepsilon})]^{2}\right) d \sigma .
$$

Here the nanotube length $l$ is constant due to the periodic boundary condition imposed along the axis of the cylinder; hence, we can consider only the strain tensor $\hat{\varepsilon}=\left(\begin{array}{ll}\zeta & 0 \\ 0 & 0\end{array}\right)$ along the circumference. So considering that $\zeta=\frac{\gamma-\gamma_{o}}{\gamma_{o}}=\frac{R-R_{o}}{R_{o}}$ :

$$
U_{s}=\frac{\pi R_{o} E l}{1-\nu^{2}}\left(\frac{R-R_{o}}{R_{o}}\right)^{2}
$$

Obviously, when $R \rightarrow R_{o}$, the stretching energy goes to zero, $U_{s}=0$.

\subsection{Step 2: Predicting the Actual Atomistic Structure of a Folded Graphene Membrane}

Tight binding (TB) atomistic simulations have been performed making use of the $\mathrm{sp}^{3}$, orthogonal, and next-neighbors tight-binding representation by $\mathrm{Xu}$ et al. [40] The present TB total energy model has been implemented within the scheme given by Goodwin et al. [41] for the dependence of the TB hopping integrals and the pairwise potential on the interatomic separation.

The following continuum analysis is useful to create reasonable input configurations for atomistic calculations, mainly with the aim of starting the relaxation routine from a configuration as close as possible to equilibrium. The investigated system consists in a squeezed nanotube formed by a perfect hexagonal carbon lattice, having circumference $L$ and length $l$, corresponding to a simulation box containing 900 carbon atoms. Moreover, periodic boundary conditions are assumed along the direction of the length $l$. The length (width) is developed along the armchair (zig-zag) direction of the honeycomb lattice.

Although reassuring, the above picture must be refined in order to take properly into account atomic-scale features. Full relaxation of the internal degrees of freedom of the systems is performed by zero temperature damped dynamics until interatomic forces result as not larger than $\sim 10^{-5} \mathrm{eV} / \AA$. We have so generated a set of configurations, where bending and stretching features are entangled, in order to simulate the HRTEM images.

Summarizing, we started from a configuration made by a hexagonal configuration mapped on the predicted shape with the desired chirality, then we obtained the correct values for the geometrical parameters using zero-temperature atomistic relaxation simulations adopting a TB semi-empirical scheme [31] plus a van der Waals interaction [42]. If the central region, where the layers remain parallel, is large enough, as shown in Fig. 15, any further constraint is not needed. The atomic 
Fig. 19 Lateral, left, and top view, right, of the half-portion of the armchair tubular lattice structure
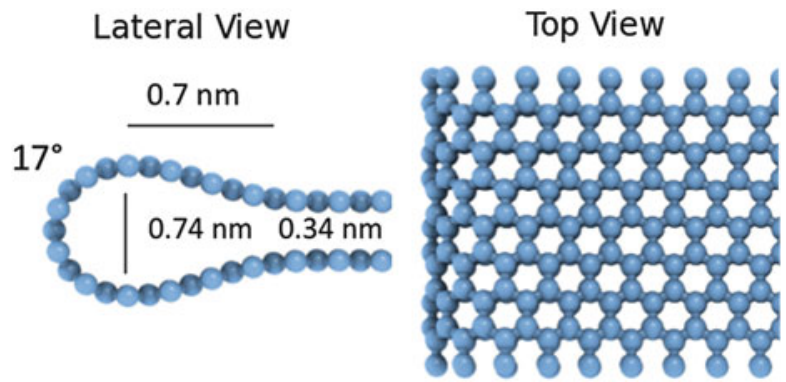

coordinates calculated so far allow us to simulate HREM images. It is important to note that in two-dimensional out-of-plane deformations it is impossible to achieve bending without introducing strain [18], and there is always interplay between real bond-length variations and the apparent strains due to the effect of projection of a bent structure. However, atomistic simulations performed on folded monolayer structures show that bond-length variations in the folded regions are $<0.1 \%$ in the direction perpendicular to the edge [18]. This indicates that graphene stiffness ensures that changes in the interatomic distances are small compared to the effect of projection on the measured strain in the image.

\section{Validation of the Experimental Procedure}

As highlighted in the previous section, the modeling of the structure started from tubular lattices, imposing the structure to collapse at the center in order to simulate the two superimposed graphenes near a folded edge, as reported in Fig. 19. In this case one half of the structure of an armchair tube has been taken into account prolonged with flat graphenes, obtaining a folded monolayer edge, with a loop of $0.74 \mathrm{~nm}$ in diameter. The height variation with respect to the center of $0.2 \mathrm{~nm}$ is accommodated over a length of $0.7 \mathrm{~nm}$ with a slope angle of $17^{\circ}$. It is important to note that variations of the interatomic distances in the curved part result in being $<1 \%$, and therefore all the strain in a TEM image of such a structure should come from the effect of the projection of the atomic positions in the lattice.

The atomic position of the lattice obtained in this way has then been used as input to simulate HREM images, in exactly in the same experimental conditions shown before, using the JEMS package [43]. In Fig. 20a the top view of the folded armchair structure is given, while in Fig. 20b the corresponding simulated HREM image is shown for an energy of $100 \mathrm{keV}$ and a small positive defocus and slightly positive spherical aberration. Then we applied the GPA to the simulated image to calculate the strain in the direction parallel to the border and perpendicular to it, as reported in Fig. 20c, d, respectively. In the direction parallel to the border there is no variation in the lattice, as we expect from the folding, where all the compression in the sloped region is along the perpendicular direction. 
a

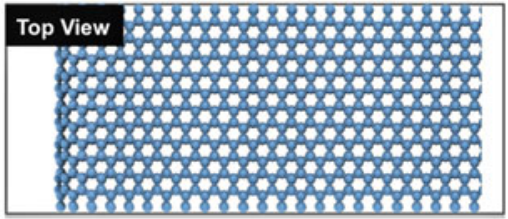

b

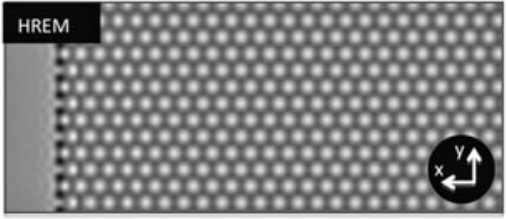

C

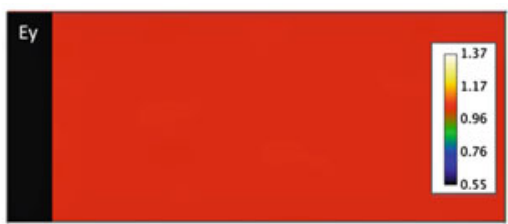

d

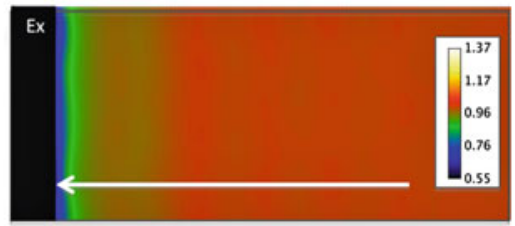

Fig. 20 (a) Top view of the simulated atomic position; (b) simulated HREM image from (a); (c) strain map along the direction parallel to the border; (d) strain map along the direction perpendicular to the border

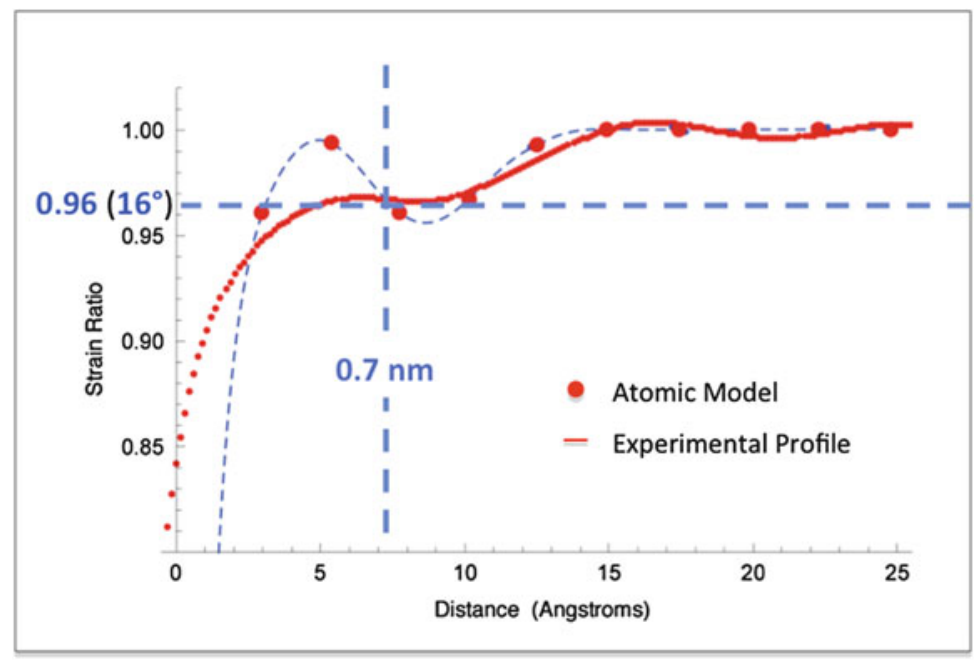

Fig. 21 Strain profile (red line) along the white line in Fig. 20d and compression values (big red dots) calculated from the projected atomic position of the simulated structure of Fig. 19

In Fig. 21 the line scan taken in the direction perpendicular to the folded border, along the white line in Fig. 20d, is shown. Moving from the flat region towards the border, we can measure a compression of about $4 \%$, followed by a rapid increase of the strain as we approach the border where the crystal is almost parallel to the beam direction.

The large red dots in the profile are the compression values calculated from the projected atomic positions in the structure of Fig. 20d. The profile maps very well the compression corresponding to the inner curvature but, due to intrinsic resolution problems in the recovered strain map, it fails to map the rapid curve of the outer 


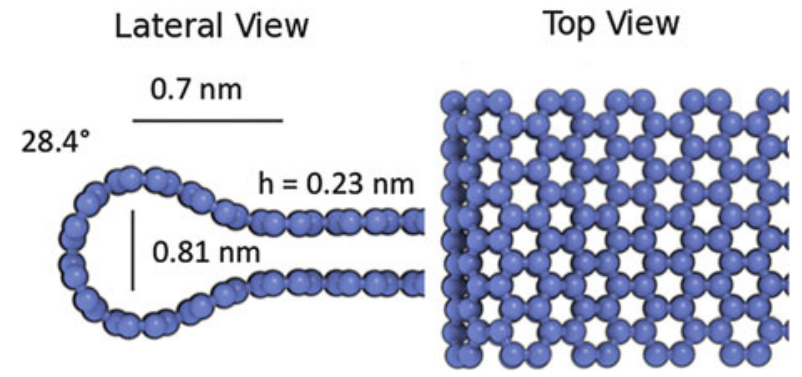

Fig. 22 Lateral, left, and top view, right, of the half-portion of the zig-zag tubular lattice structure

a

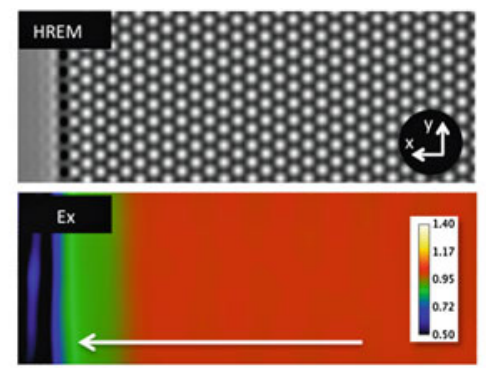

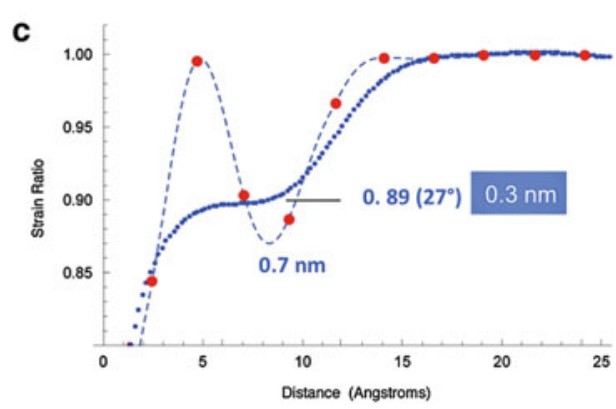

Fig. 23 (a) Simulated HREM image from the simulated atomic positions of Fig. 22; (b) strain map along the direction parallel to the border; (c) strain profile (blue line) along the white line in (b) and compression values (big red dots) calculated from the projected atomic position of the simulated structure of Fig. 22

part. Nevertheless, as in the experimental case, the intensity of the compression can be used to measure the local maximum slope, about $16^{\circ}$, and to measure the length of the curved region, estimated as $0.7 \mathrm{~nm}$. Therefore it is possible to estimate a height variation of $0.2 \mathrm{~nm}$, which is almost identical to the real height variation in the simulated structure.

To confirm further the capabilities of the method, following the very same approach, one half of the structure of a zig-zag tube has been taken into account, as reported in Fig. 22. In this case the modeled structure shows a loop of $0.81 \mathrm{~nm}$ in diameter, and thus a height variation with respect to the center of $0.23 \mathrm{~nm}$ accommodated over a length of $0.7 \mathrm{~nm}$ with a slope angle of about $28^{\circ}$. Again we can use the modeled atomic position as input for HREM image simulation to apply the GPA strain analysis to recover the 3D modeled structure. The results are reported in Fig. 23. Even in this case the spatial resolution of the map fails to show the rapid curvature at the outer edge, but at the same time it is possible to measure with great precision the structure of the internal region, with a slope of $27^{\circ}$, over a length of $0.7 \mathrm{~nm}$, therefore estimating a height variation of $0.3 \mathrm{~nm}$, close to the 0.23 expected from the model. 


\section{Conclusions}

In this chapter we have presented a novel approach to study the mechanical properties of wrinkles and fold in graphene membrane, using a combination of TEM-based 3D mapping and blended continuum-atomistic modeling. The experimental results show that the apparent strain in the HREM images on graphene membranes provides precise information about the 3D sub-nanometer height and spatial resolution, in excellent agreement with predictions by atomistic tight-binding simulations.

Combining information from electron diffraction and HREM with the possibility of mapping the 3D membrane morphology is successful in characterizing freely suspended graphene crystals. In this work we have focused on the investigation of graphene folded edges, but the same methodology can be applied to investigate the elastic properties and 3D structure of complex folds and wrinkle geometries. In addition, the proposed approach is general and can be easily extended to other two-dimensional crystal like $\mathrm{BN}$ or $\mathrm{MoS}_{2}$ membranes, as well as to hybrid multilayer thin-films composed of these materials.

Acknowledgement One of us (L.C.) acknowledges financial support under project PRIN 2010-2011 "GRAF".

\section{References}

1. Krivanek OL, Dellby N, Murfitt MF, Chisholm MF, Pennycook TJ, Suenaga K, Nicolosi V (2010) Gentle STEM: ADF imaging and EELS at low primary energies. Ultramicroscopy 110:935-945

2. Warner JH, Roxana Margine E, Mukai M, Robertson AW, Giustino F, Kirkland AI (2012) Dislocation-driven deformations in graphene. Science 337:209-212

3. Meyer JC, Eder F, Kurasch S, Skakalova V, Kotakoski J, Jin Park H, Roth S, Chuvilin A, Eyhusen S, Benner G, Krasheninnikov AV, Kaiser U (2012) Accurate measurement of electron beam induced displacement cross sections for single-layer graphene. Phys Rev Lett 108:196102

4. Zan R, Bangert U, Ramasse Q, Novoselov KS (2011) Metal-graphene interaction studied via atomic resolution scanning transmission electron microscopy. Nano Lett 11:1087-1092

5. Zhou W, Kapetanakis MD, Prange MP, Pantelides ST, Pennycook SJ, Idrobo JC (2012) Phys Rev Lett 109:206803

6. Williams DB, Carter CB (2009) Transmission electron microscopy: a textbook for materials science. Springer, New York

7. Pennycook SJ, Nellist PD (2011) Scanning transmission electron microscopy. Springer, New York

8. Hytch M, Snoeck E, Kilaas R (1998) Quantitative measurement of displacement and strain fields from HREM micrographs. Ultramicroscopy 74:131-146

9. Grillo V, Rossi F (2013) Stem cell: a software tool for electron microscopy. Part 2. Analysis of crystalline materials. Ultramicroscopy, advanced online publication 125:112-129

10. Mermin N (1968) Crystalline order in two dimensions. Phys Rev 176:250-254

11. Novoselov K, Jiang D, Schedin F, Booth T, Khotkevich V, Morozov S, Geim A (2005) Two-dimensional atomic crystals. Proc Nat Acad Sci USA 102:10451-10453 
Folds and Buckles at the Nanoscale: Experimental and Theoretical...

12. Meyer JC, Geim AK, Katsnelson MI, Novoselov KS, Booth TJ, Roth S (2007) The structure of suspended graphene sheets. Nature 446:60-63

13. Bao W, Miao F, Chen Z, Zhang H, Jang W, Dames C, Lau CN (2009) Controlled ripple texturing of suspended graphene and ultrathin graphite membranes. Nat Nanotech 4:562-566

14. Vandeparre H, Pineirua M, Brau F, Roman B, Bico J, Gay C, Bao W, Lau CN, Reis PM, Damman P (2011) Wrinkling hierarchy in constrained thin sheets from suspended graphene to curtains. Phys Rev Lett 106:224301

15. Kim K, Lee Z, Malone BD, Chan KT, Aleman B, Regan W, Gannett W, Crommie MF, Cohen ML, Zettl A (2011) Multiply folded graphene. Phys Rev B 83:245433

16. Topsakal M, Bagci VMK, Ciraci S (2010) Current-voltage (I-V) characteristics of armchair graphene nanoribbons under uniaxial strain. Phys Rev B 81:205437

17. Cadelano E, Palla P, Giordano S, Colombo L (2009) Nonlinear elasticity of monolayer graphene. Phys Rev Lett 102:235502

18. Cadelano E, Giordano S, Colombo L (2010) Interplay between bending and stretching in carbon nanoribbons. Phys Rev B 81:144105

19. Poetschke M, Rocha CG, Foa Torres LEF, Roche S, Cuniberti G (2010) Modeling graphenebased nanoelectromechanical devices. Phys Rev B 81:193404

20. Feng J, Qi L, Huang J, Li J (2009) Geometric and electronic structure of graphene bilayer edges. Phys Rev B 80:165407

21. Tozzini V, Pellegrini V (2011) Reversible hydrogen storage by controlled buckling of graphene layers. J Phys Chem C 115:25523-25528

22. Zheng Y, Wei N, Fan Z, Xu L, Huang Z (2011) Mechanical properties of grafold: a demonstration of strengthened graphene. Nanotechnology 22:405701

23. Prada E, San-Jose P, Brey L (2010) Zero landau level in folded graphene nanoribbons. Phys Rev Lett 105:106802

24. Zhu W (2012) Structure and electronic transport in graphene wrinkles. Nano Lett 12:3431-3436

25. Pang ALJ, Sorkin V, Zhang Y-W, Srolovitz DJ (2012) Self assembly of free-standing graphene nano-ribbons. Phys Lett A 376:973-977

26. Qi L, Huang JY, Feng J, Li J (2010) In situ observations of the nucleation and growth of atomically sharp graphene bilayer edges. Carbon 48:2354-2360

27. Patra N, Wang B, Kral P (2009) Nanodroplet activated and guided folding of graphene nanostructures. Nano Lett 9:3766-3771

28. Catheline A, Ortolani L, Morandi V, Melle-Franco M, Drummond C, Zakri C, Penicaud A (2012) Solutions of fully exfoliated individual graphene flakes in low boiling points solvents. Soft Matter 8:7882-7887

29. Midgley P, Weyland M, Yates T, Tong J, Dunin-Borkowsky R (2004) Stem electron tomography for nanoscale materials science. Microsc Microanal 10:148-149

30. Molhave K, Gudnason SB, Pedersen AT, Clausen CH, Horsewell A, Boggild P (2007) Electron irradiation-induced destruction of carbon nanotubes in electron microscopes. Ultramicroscopy 108:52-57

31. Barboza APM, Chacham H, Oliveira CK, Fernandes TFD, Martins Ferreira EH, Archanjo BS, Batista RJC, de Oliveira AB, Neves BRA (2012) Dynamic negative compressibility of few-layer graphene, h-BN, and $\mathrm{MoS}_{2}$. Nano Lett 12:2313-2317

32. Hytch M (1997) Analysis of variations in structure from high resolution electron microscope images by combining real space and Fourier space information. Microsc Microanal 8:41-57

33. Hytch M, Plamann T (2001) Imaging conditions for reliable measurement of displacement and strain in high-resolution electron microscopy. Ultramicroscopy 87:199-212

34. Snoeck E, Warot B, Ardhuin H, Rocher A, Casanove M, Kilaas R, Hytch M (1998) Quantitative analysis of strain field in thin films from HRTEM micrographs. Thin Solid Films 319:157-162

35. Ortolani L, Cadelano E, Veronese GP, Degli Esposti Boschi C, Snoeck E, Colombo L, Morandi V (2012) Folded graphene membranes: mapping curvature at the nanoscale. Nano Lett 12:5207-5212 
36. Xu CH, Wang CZ, Chan CT, Ho KM (1992) A transferable tight-binding potential for carbon. J Phys Cond Matt 4:6047-6054

37. Dresselhaus G, Saito R, Dresselhaus MS (1998) Physical properties of carbon nanotubes. Imperial College, London

38. Shen L, Li J (2005) Equilibrium structure and strain energy of single-walled carbon nanotubes. Phys Rev B 71:165427

39. Chang T, Gao H (2003) Size-dependent elastic properties of a single-walled carbon nanotube via a molecular mechanics model. J Mech Phys Sol 51:1059

40. Xu Y, Gao H, Lil M, Guo Z, Chen H, Jin Z, Yu B (2011) Electronic transport in monolayer graphene with extreme physical deformation: ab initio density functional calculation. Nanotechnology 22:365202

41. Goodwin L, Skinner AJ, Pettifor DG (1989) Generating transferable tight-binding parameters: application to silicon. Europhys Lett 9:701-706

42. Rappe AK, Casewit CJ, Colwell KS, Goddard WA III, Skiff WM (1992) UFF, a full periodic table force field for molecular mechanics and molecular dynamics simulations. J Am Chem Soc 114:10024-10035

43. JEMS P Stadelmann, CIME-EPFL, Laudanne, Switzerland. http://cimewww.epfl.ch/people/ stadelmann/jemsWebSite/jems.html. Accessed online 2013 